\title{
Semiotik
}

Band $36 \cdot$ Heft 3-4 (2014)

Seite 141-186

Stauffenburg Verlag Tübingen

\section{Löst Brandoms Inferentialismus bedeutungsholistische Kommunikationsprobleme?}

\author{
Axel Mueller, Northwestern University
}

\begin{abstract}
Summary. This article analyzes whether Brandom's ISA (inferential-substitutional-anaphoric) semantics as presented in Making It Explicit (MIE) and Articulating Reasons (AR) can cope with problems resulting from inferentialism's near-implied meaning holism. Inferentialism and meaning holism entail a radically perspectival conception of content as significance for an individual speaker. Since thereby its basis is fixed as idiolects, holistic inferentialism engenders a communication problem. Brandom considers the systematic difference in information among individuals as the „point" of communication and thus doesn't want to diminish these effects of inferentialism. Instead, explains communication with a model of "navigating among perspectives without sharing contents". The crucial element in this navigation-model is the functioning of anaphoric connections between tokens uttered in discourse that can be used by every individual speaker in their own perspectival semantic substitution-economies. The heart of Brandom's semantics is the thesis of the purely inferential, hence non-referential nature of anaphora, coupled with the claim that anaphoric-inferential semantic mechanisms yield sufficient conditions for mutually successful "information-extraction" or interpretation. This article disputes the thesis and denies the claim. Regarding the former it is observed that all of Brandom's plausible reconstructions of anaphoric discourse-structures rely on covert "reference-infiltrations" that can't be eliminated. Regarding the latter, a new argument based on context-sensitive semantic phenomena in anaphoric settings shows that the crucial distinction between initiator or anaphoric antecedent and anaphoric dependent cannot be drawn according to Brandom's own premises without overt and irreducible referential premises. The article concludes that either Brandom's semantics can offer determinate contents, but then must accept genuinely referential semantic primitives, or else it leaves utterance-contents undeterminable and hence cannot explain communication.
\end{abstract}

Zusammenfassung. Dieser Beitrag untersucht, inwieweit Brandoms in Making It Explicit (MIE) und Articulating Reasons (AR) entwickelte inferentialistische Semantik Probleme lösen kann, die sich aus dem im Inferentialismus unter relativ unkontroversen Zusatzannahmen entstehenden und von Brandom bewusst zugrundegelegten Bedeu- 
tungsholismus ergeben. Aus dem Inferentialismus folgt mit Bedeutungsholismus eine perspektivische Bestimmung sprachlichen Gehalts, die wegen der damit angenommenen Idiolektbasis ein Kommunikationsproblem erzeugt. Brandom betrachtet dieses systematische Informationsgefälle als natürliche Motivation zur Kommunikation. Er erklärt Kommunikation mittels eines Modells des „Navigierens zwischen Perspektiven“, das letztlich auf der Verfügbarkeit substitutioneller und anaphorischer Äußerungsverknüpfungen beruht. Semantisch entscheidend ist die These, dass anaphorische Ketten rein inferentieller, also referenzfrei semantisch bestimmter Natur sind, sowie die Behauptung, dass anaphorisch-inferentielle Mittel zu gegenseitig erfolgreicher „Informationsextraktion“ oder „Interpretation“ hinreichen. In diesem Aufsatz wird die These bestritten. Erstens lassen sich in Brandoms plausiblen Darstellungen anaphorischer Kommunikationsstrukturen systematische „Referenzinfiltrationen“ nachweisen. Dass dies die Behauptung unterminiert zeigt sich zweitens an einer Analyse der Durchführbarkeit einer semantisch entscheidenden inneranaphorischen Unterscheidung (zwischen Initiator und Glied) im Rahmen kontextsensitiver Ausdrucksformen. Brandoms Semantik kann entweder für Sprecher und Hörer bestimmbare Gehalte annehmen, muss aber dann irreduzibel referenzielle semantische Normen unterstellen, oder aber sie lässt Gehalte systematisch unbestimmbar.

\section{Einleitung}

In diesem Beitrag möchte ich untersuchen, inwieweit Brandoms in Making It Explicit (MIE) und Articulating Reasons (AR) entwickelte und in Between Saying and Doing (BSD) weiterentwickelte inferentialistische Theorie sprachlicher Bedeutung oder des Gehalts sprachlicher Äußerungen die sich selbst gestellten Probleme lösen kann. Die fraglichen Probleme ergeben sich aus dem im Inferentialismus unter relativ unkontroversen Zusatzannahmen entstehenden und von Brandom bewusst zugrundegelegten radikalen Bedeutungsholismus. Solche Probleme, die im Verlauf der Weiterentwicklung von Brandoms Position immer mehr in den Mittelpunkt rücken, erklärt er wiederholt explizit als lösbar durch die spezifischen Mittel seiner inferentialistischen Position. Angesichts der vielfältigen Einwände, die gegen die holistische Implikationen seiner Theorie vorgebracht worden sind, stellt Brandom diese Lösung durch die spezifischen Mittel seiner Position in zunehmendem Maße als eines der Kernverdienste seiner speziellen inferentialistischen Theorie in den Vordergrund. ${ }^{1}$

Das fundamentale Problem für einen bedeutungsholistischen Inferentialismus ist Brandoms eigener Analyse nach schnell erzählt. Wird Signifikanz oder Gehalt $^{2}$ von verwendeten Ausdrücken, Gedanken und Handlungen inferentialistisch als Rolle in einem gegebenen Folgerungssystem charakterisiert, aber zugleich bedeutungsholistisch keine noch so wahrnehmungsrelative Festlegung oder Folgerung eines kognitiven Systems von den potentiell gehaltsbestimmenden Bedingungen ausgeschlossen, dann stellt sich unter der unproblematischen Annahme, dass die genauen Hintergrundbedingungen zur Gehaltsbestimmung individuell variieren werden, die unmittelbare Frage, wie Kommu- 
nikation unter dieserart perspektivisch gehaltsbestimmenden Individuen möglich ist. ${ }^{3}$ Dies nenne ich im Folgenden das Ko m mu n ikation sp roble m.

Brandoms Grundhypothese ist, dass seine auf einer we it e n Basis verfahrende inferentialistische Position (AR:28f.), die praktische Folgerungen und Sprecherhandlungskontexte einschließt und die erweitert ist um anaphorischsubstitutionelle sowie weitere induktive Schlussregeln, dieses Problem lösen kann. Seine weitergehende These ist, dass die Theorie es dabei vermeiden kann, auf den inferentialistischen Rahmen sprengende Grundbegriffe aus dem repräsentationalist is chen Repertoire (nämlich die Bezugnahme auf außersprachliche Gegenstände als unabhängig semantisch kodifizierte Leistung und Korrektheitsbedingung von Behauptungen) zurückzugreifen. Erfolgreiche Kommunikation über Unterschiede in inferentiell durch Kohärenznormen vollauf bestimmten und gehaltsbestimmenden Überzeugungssystemen hinweg, soll resolut sozial-inferentialistisch erklärt werden. Brandoms Ansatz ist so - malgré lui même - eher intellektualistisch als pragmatistisch. ${ }^{4}$

Brandoms wiederholte Insistenz auf eine gebrauchstheoretische Strategie, dass "semantics must [...] answer pragmatics" (AR: 125) steht damit keineswegs in Spannung. Sie heißt nur, dass allein der Rückgriff auf implizit normative Sprachgebrauchsmuster in einer Sprachgemeinschaft (use) legitime Ressourcen für semantische Theoriebildung (meaning) darstellt. Anderen Ressourcen wie etwa die der interaktiven Handlungstheorie und den darin zugrunde gelegtengemeinsamen und gegenseitig zugeschriebenen Weltbe zügen (etwa im Know-how) darf dagegen keine legitime Grundlagenfunktion für die Erklärung semantischer Phänomene zukommen. Sein Ansatz, "offering an account of referential relations to objects in terms ultimately of inferential relations among claims", beansprucht mittels Durchführung den Beweis zu führen, dass "inferential articulation broadly construed is sufficient to account for conceptual content" (AR:28). Ohne Umsetzung praktischer Bezüge in inferentiell verflechtbare und in Zuweisungen epistemischer Status resultierende Behauptungen können auch Interaktionen und Weltbezüge diesem Prinzip nach keine Erklärungslast tragen: "eschew representational primitives" (MIE: 132). Im Folgenden nenne ich dies Brandoms Ver m e i du n g s p ri nzip.

Dieser Aufsatz soll begründen, dass die Analyse der Bedingungen der erfolgreichen Verwendung anaphorischer Mittel (und damit die von Brandom als lediglich inferentiell aber nicht referenziell deklarierte Hinzufügung zum inferentialistischen Apparat) zeigt, dass weder anaphorisch-substitutionelle noch die Kombination anaphorisch-substitutioneller Verfahren mit kausalen Umwelteinbettungen des Sprachgebrauchs ohne ein Äquivalent des als "different primitive" (MIE: 481f.) abgelehnten Referenzbegriffs für Kommunikationszwecke ausreichend wohlbestimmt sind. ${ }^{5}$ Meine Argumentation folgt Brandoms eigener Darstellung, aber verschärft einige Aspekte darin, indem sie dem Brandoms Inferentialismus eigenen Erklärungsziel - der Erklärung von erfolgreicher Kommunikation - nachgeht und dabei die Einhaltung des Referenzvermeidungsprinzips strikt überwacht. ${ }^{6}$ Das spitzt, wie bereits 
anderweitig bemerkt (Laurier 2008; Prien 2010; Whiting 2009; Loeffler 2009; Wanderer 2008), das Kommunikationsproblem perspektivistisch zu. ${ }^{7}$

Zur Entwicklung dieses Arguments werde ich zunächst kurz das Kommunikationsproblem skizzieren (Kapitel 1). Im Anschluss werde ich Brandoms Ablehnung verschiedener Lösungsmöglichkeiten ansprechen, die entgegen der Perspektivenrelativität inferentialistisch definierter Signifikanzen auf perspektivenübergreifend geteilte Hintergrundannahmen verweisen. Brandom verfolgt stattdessen die Idee der unabhängig von der Übernahme der inferentiellen Festlegungen stattfindenden Ver e rbung des Gehalts von Äußerungen (Kapitel 2). Diese Idee wird üblicherweise mittels repräsentationalistischer Grundbegriffe erläutert wie die durch Überzeugungsdifferenzen invariant bleibenden Bezüge sprachlicher Zeichen auf als Gehalt festgelegte, überzeugungsunabhängig Sprechern und Hörern verfügbare Gegenstände. Brandom schlägt dagegen vor, die Verfügbarkeit von externen Bezügen mittels der gemeinsamen Einbettung inferentieller und diskursiver Praktiken in eine Umwelt und der pragmatistischen Idee zu rekonstruieren, dass das inferentielle Repertoire w e it ge fa s st genug ist, um auch aus Wahrnehmungen erworbene unwillkürliche Überzeugungen als inferentielle Eingangsbedingungen und Handlungen in der Umwelt als Ausgangsbedingungen zu rekonstruieren. Die Ve re rbung von perspektivenneutralem Gehalt ließe sich statt durch die Bezugnahme auf nichtperspektivische Gegenstände durch das Bestehen eines perspektivenneutralen Weitergabemechanismus, eben der Anapher, als zusätzlicher inferentieller Ressource zur Ersetzung von Sprecher- durch Hörerausdrücke erläutern. Ich werde daher zunächst (Kapitel 3) diskutieren, ob sich aus der We itge fasstheit des Inferenzbegriffs ohne implizite Inanspruchnahme referenzieller Normen kognitiv effektive Gemeinsamkeiten der Teilhabe an Umwelt ergeben, die einen eigenständigen Beitrag zur Lösung des Kommunikationsproblems leisten. Es wird sich erweisen, dass für die Herstellung ge meins a m e $r$ inferentiell relevanter Umweltbedingungen entweder nichtperspektivische semantische Normen angenommen werden müssen oder die Gemeinsamkeit der Umwelt keine bestimmten Gehaltsnormierungen leistet. Im Anschluss an diesen Nachweis der Abhängigkeit des Beitrags der Umwelt zur Lösung des Kommunikationsproblems von nichtperspektivischen semantischen Normen (die ich als illegitime Referenzinfiltration bezeichne) stelle ich dann Brandoms solche Normen bereitzustellen beanspruchendes Modell der Anapher als Lösungsvorschlag dar (Kapitel 4). Diesem Modell zufolge ist die Bestimmtheit anaphorisch ve re rbte $n$ Gehalts abhängig von den die Kette initiierenden Äußerungen, an die alle weiteren Kettenglieder gehaltserhaltend anknüpfen. Ich werde im Anschluss darstellen, dass die Unters c h e i d ung zwischen Initiatoren und Gliedern selbst Fähigkeiten von Sprechern erfordert, die sich nicht in ihren Fähigkeiten zur gehaltserhaltenden Anknüpfung erschöpfen können. Daher bleiben referenzfrei generierte beziehungsweise beschriebene anaphorische Ketten se mantis $\mathrm{ch}$ unbestim mbar und können aus diesem Grund nicht für die Lösung des Kommunikationsproblems hinreichen (Kapitel 5). Den Nachweis für die Abhängigkeit der Unterscheidung von Initiator und Kettengliedern von spezifisch r e fe - 
renziellen Normen führe ich daran anschließend mit Bezug auf verschiedene Phänomene kontextabhängiger semantischer Bestimmtheit (Kapitel 6, 7). Meine Schlussfolgerung ist, dass Brandoms als referenzanalog angeführtes Modell der Gehaltsvererbung bei strikter Befolgung des Referenzvermeidungsprinzips kein Modell der Vererbung bestim m te $r$ Gehalte darstellt und deswege n keine Lösung des Kommunikationsproblems leisten kann. Ein anaphorisches Modell kann aber eine solche Erklärung auf p ra g m a t i scher Basis leisten, wenn es mit spezifisch referenziellen Normen als eigenständigen Zusatzressourcen angereichert wird. Hält man das Vermeidungsprinzip aufrecht, so opfert man die Lösung des Kommunikationsproblems (und aller darauf aufbauenden Erklärungsleistungen). Opfert man das Vermeidungsprinzip, löst man unter Zuhilfenahme referenzieller Normen das Kommunikationsproblem. Für letzteres wird weder der Holismus noch die inferentialistische Erklärung des kognitiven Werts sprachlicher Ausdrücke eingeschränkt. Ich sehe dies als gute Gründe für die Hypothese der I r r e d u zibilität substantiell referenzieller (abernicht naturalistisch auf Kausaltheorien verkürzter) Elemente für eine den Anforderungen kommunikativ erfolgreicher behauptender Rede adäquate semantische Theorie an. Deswegen scheint mir die zweite (von Brandom selbst stellenweise suggerierte) Weiterentwicklung für mit Brandoms Ansatz sympathisierende Weiterentwicklungen vorzuziehen. ${ }^{8}$

\section{Das Kommunikationsproblem in Brandoms Inferentialismus und Bedeutungsholismus}

Dass der im Inferentialismus entwickelte Begriff sprachlich kodierten Gehalts we sentlich holistisch ist (und eine inferentialistische Bedeutungstheorie somit typischerweise holistisch ist) betont Brandom vielerorts, wie zum Beispiel in AR, wo es heißt:

"inferentialist semantics is resolutely holist. On an inferentialist account of conceptual content, one cannot have any concepts unless one has many concepts. For the content of each concept is articulated by its inferential connections to other concepts. [...] Conceptual holism is [...] a straightforward consequence of this approach" (AR: 15f.).

Konkreter im Rahmen von Brandoms pragmatisch-inferentialistischem Ansatz resultiert der Holismus aus den beiden Entscheidungen, dass es erstens Aufgabe der Semantik ist, eine Theorie des kognitiven oder durch Verstehen sprachlicher Handlungen sich ergebenden Gehalts (oder des Fregeschen S in n s) sprachlicher Ausdrücke zu liefern. Zweitens dafür, dass dieser Gehalt als die sich im Geben und Fordern von Gründen kommunikativ einstellende Gesamtheit der Schlussfolgerungen zu konzipieren ist, die sich für einen Hörer aus der kritischen Prüfung der Auf- oder Übernahme einer Sprecherbehauptung als Prämisse oder Konklusion in ein ihm als Hintergrundannahmen kognitiv verfügbares System von propositionellen Gehalten ergeben. Dies zieht nach sich, 
dass der verstandene Gehalt eines Ausdrucks nur im Zusammenhang der sich für das Gesamtsystem ergebenden folgerungsrelevanten kognitiven Konsequenzen bestimmt werden kann. Dass ein sprachlicher Ausdruck überhaupt irgendeinen Gehalt hat, setzt laut Brandom voraus, dass er eine Rolle als Prämisse oder Konklusion in einer Behauptungen als Gründe oder Folgerungen austauschenden sozialen Praxis (kognitiver Kommunikation) spielen kann oder (im Falle subsententieller Ausdrücke) einen systematischen Beitrag zu einer solchen Rolle leistet. Kein Gehalt ohne Verwendbarkeit in einer Behauptungspraxis. Welchen Gehalt ein bestimmter Ausdruck (im Gegensatz zu anderen gehaltvollen Ausdrücken ähnlicher grammatischer Art) hat, ergibt sich wiederum vollständig aus der Rolle, die der Ausdruck für das Gesamtsys tem guter Schlussfolgerungen spielt, dem er angehört.

Dass der Bedeutungsholismus den Inferentialismus in eine Zwangslage bringt, stellt Brandom bereits in MIE heraus, denn "inferential significance is not preserved in communication", während der Rückgriff auf inferenzu nabhängige Bezugnahme auf dasselbe über Unterschiede in (inferentiell durch Kohärenznormen determinierten) Überzeugungsgesamtheiten hinweg "gives up on inferential roles, in favor of a different sort of primitive" (MIE: 480ff.). Brandoms Erklärung der Nichterhaltung inferentieller Signifikanz zwischen Sprecher und Hörer geht zwar zunächst konkret von Freges Analyse von Schlüssen mit mehreren Prämissen aus, hätte aber auch verwandte Gedanken von Duhem oder Quine zur Unabgeschlossenheit der Falsifikation nehmen können (wie an späterer Stelle in MIE und in späteren Veröffentlichungen (MIE: 477ff.; Brandom 1997; 2010)). Das Grundproblem ergibt sich, wenn die A n we n dungskorrektheit einer gegebenen Behauptung sensitiv auf Veränderungen der Prämissenmenge (das heißt der verfügbaren Information) reagiert.

Beispielsweise folgt aus der Sprecherinnenbehauptung Vögel fliegen für die mit heimischen Singvögeln, Möwen und Reihern vertraute Schleswig-Holsteiner Hörerin, dass der vor ihr sitzende Eike, der riesige Elsterhahn, da er Vogel ist, fliegt (fliegen wird), selbst wenn er wegen eines gebrochenen Flügels gerade nicht fliegen kann. Die Behauptung Eike fliegt folgt allerdings nicht für eine zweite Hörerin, die aufgrund des Aussehens von Eike und der i h r, aber nicht der ersten Hörerin verfügbaren Information Pinguine sind Vögel aus derselben wahrgenommenen Szene schließt Eike ist ein elsternartig aussehender Pinguin und wegen der daraus folgenden Flugunfähigkeit von Eike schließt, dass Eike auch nach Abheilen des Flügelbruchs nicht fliegt. Für wahrnehmungs- und anderweitig gleich situierten Sprecherinnen ergeben sich also aus Vögel fliegen unterschiedliche inferentielle Signifikanzen und unterschiedliche empirische Folgerungen ( E i ke fliegt ( $\mathrm{nicht}$ )) aufgrund der Unterschiede in kollateralen Begleitfestlegungen. Wie Brandom sagt: "a single commitment typically has a different significance for $[\ldots]$ a speaker [...] from that which it would have for [...] an audience or [...] interpreter" (MIE: 139). Während diese augenscheinliche Informationsdifferenz hinsichtlich derselben Aussage zwanglos erklärt, weswegen Menschen überhaupt an Kommunikation interessiert sind - nämlich um die anderen verfüg- 
baren Sichtweisen und damit verbundenen anderen Informationspotentiale zu erhalten - ergibt sich vor den so soziale Praxis rationalisierenden Vorteilen der Kommunikation das p rima fa cie Problem, wie Kommunikation überhaupt als Informationsaustausch möglich ist. Brandom definiert in MIE die Bedingung für die Übermittelbarkeit von Information so:

"For information (whether true or false) to be communicated is for the claims undertaken by one interlocutor to become available to others (who attribute them) as premises for inferences. Communication is the social production and consumption of reasons" (MIE: 474).

Dafür müssen die Gesprächsteilnehmer allerdings e rst e in mal die Verlautbarungendesanderen als gehaltvoll undals bestimmte Gehalte enkodierend identifizieren, und das ist mit dem bedeutungsholistischen Gehaltbegriff nicht von selbst erklärt, da sich ja die Signifikanzen p e r s p e k t i ve n relativ auf die individu ell lokalisierten unterschiedlichen Hintergrundrepertoires bestimmen. Brandom formuliert diesen Umstand deutlich als die Ansicht, dass der inferentialistische Ansatz "the perspectival nature of propositional contents" behauptet (MIE: 485). Während es von einem gebrauchstheoretisch und betont pragmatistischen Ansatz wie Brandoms vielleicht zu erwarten wäre, dass von einer genuin sozialen und durch gemeinsame Teilhabe an Gehalten charakterisierbaren Basis ausgegangen wird, überrascht es vor diesem Hintergrund - im Gegensatz zu einer herkömmlichen Lesart des Sozialen als auf gegenseitig bewusste oder befolge Gemeinsamkeiten gegründeter Zusammenarbeit - nicht, wenn Brandom direkt im Anschluss an die das Kommunikationsproblem und den damit drohenden Solipsismus auf den Punkt bringenden Feststellung der prinzipiell subjektiven Verstehensbasis de $\mathrm{fi}$ $\mathrm{nie} \mathrm{rt}$, dass dies "means that the inferential articulation of conceptual contents has a fundamental social dimension", denn diese Schlussfolgerung "introduces a relativity to [...] perspective into the specification of such contents" (MIE: 139; Hervorhebung A.M.).

Für Brandom ist Perspektivität das We s e n t l i c h e der Sozialität, von dem aus sein Ansatz (auch) eine Begründung sozialer Normen mittels der Analyse der Dynamik von Statusanpassungen gegenseitig kontoführender Teilnehmer an behauptende Begründungspraktiken zu liefern beansprucht. Weil der Informationsbedarf und das zwischen Individuen immer bestehende Informationsg e fäll e das eigentliche rationale Grundmotiv epistemischer Vergemeinschaftung darstellt, ka n n also die Grundlage von gegenseitigem Verstehen nicht in der semantischen Assoziierung von allen Sprechern gleichermaßen durch sprachliche Zeichen verfügbarer und als fixierte Be de utung zirkulierender Information gesucht werden. Brandom motiviert diesen perspektivistischen Ausgangspunkt mit zwei weiteren Betrachtungen. Die erste schließt an Quines Argumente gegen die analytisch-synthetisch Unterscheidung an. Die Postulierung perspektiveninvarianter feststehender Bedeutungen wäre inferentialistisch als die Fixierung bestimmter Folgerungen als Systeme „kanonischer Verifikationen“ (Dummett, 1991, 313f.) zu verstehen (also etwa der Festlegung 
auf für Voge/ wesentliche Eigenschaftszuschreibungen als unter keinen hinzukommenden Festlegungen falsifizierbar). Für deren Individuierung und Immunisierung gegen die oben angesprochenen Effekte von Kollateralannahmen bedürfte es eines semantischen Grundbegriffs, der inferentieller Verwendung der so fixierten Ausdrücke vorgeordnet ist und mit dem diese Behauptungsmengen ausgezeichnet werden könnten, etwa als bedeutungskonstitutiv oder analytisch. Dies verstieße aber gegen die Ablehnung der Hinzunahme extrainferentieller Grundbegriffe, denn der bedeutungskonstitutive von anderen Überzeugungen unterscheidende Begriff ist ja selbst semantisch aber der inferentiellen Verarbeitung von Begriffen als regelgenerierende Bedeutungsspezifikation vorgeordnet.

Die komplementäre zweite Betrachtung schließt an Sellars Erweiterung des inferentiellen Repertoires auf „materiale Schlussregeln“ an (MIE: 97ff.), die den Erwerb wahrnehmungsbedingter „unmittelbarer doxastischer Festlegungen“ im praktischen Umgang mit der Umwelt als inferentielle Eingangsbedingungen den Prämissen hinzufügt und Handlungen als intelligente aus informationsverarbeitienden Schlussfolgerungen hervorgehende Interventionen in die Umwelt den Konklusionen hinzufügt. Daraus ergibt sich ein we i t e $r$ Inferenzbegriff. Ist Gehalt also als inferentielle Rolle zu verstehen, dann kommt materialen, das heißt kontingent informationstragenden Verbindungen a priori genau derselbe Status als Teil des inferentiellen Systems zu wie innersprachlichen Schlüssen. Somit sind empirische Erfahrungen als potentiell bedeutungsspezifikationsrelevant nicht ohne willkürliche Inferenzblockierungen ausgeschlossen.

Aus beiden Erwägungen zusammen mit der Rationalitätsannahme über das Grundmotiv der Kommunikationspraxis ergibt sich, dass die Absonderung vorgeblich bedeutungskonstitutiver, perspektivenneutraler oder -invarianter und dann sozial als sprachkompetenzkonstitutive Untermengen aus dem inferentiellen System aussichtlos ist. Der Bedeutungsholismus führt unter inferentialistischen Prämissen also unweigerlich in ein $\mathrm{Ko}$ m m u n i k a t i o n s problem beziehungsweise ein Perspektivitäts problem des Gehalts. Wie Scharp treffend zusammenfasst, "on Brandom's model, there is no perspective-independent thing - the content of the sentence - that [...] both grasp" (Scharp 2012: 108). Wie lässt sich aber die für miteinander kommunizierende Gesprächsteilnehmer charakteristische Koordinierung durch semantische Verknüpfung kognitiver Zustände ohne die Annahme beiden verfügbarer Bedeutungen erklären?

\section{Rückzug auf die Referenz als Strategie, Navigation durch Perspektiven als Modell}

Wie Brandom selbst ausführt, ist die aus der semantischen Tradition naheliegendste direkte Antwort die, die fundamentale Bedeutungseigenschaft von in Behauptungen verwendeten sprachlichen Zeichen nicht weiter in den Differenzierungen des kognitiven Werts (also des Fregeschen Sinns), son- 
dern in ihrer Referenz beziehungsweise Extension zu suchen. Wie Brandom feststellt, "an important motivation for the emphasis on [...] the referential dimension of discourse can be found in the concern with the [...] possibility of communication" (MIE: 481). Die hierbei zum Tragen kommende Grundidee erfüllt die vom Perspektivismusproblem aufgeworfenen Anforderungen, indem sie die der Sprecherin und Hörerin gemeinsame semantische Norm als die erfolgreiche Bezugnahme auf ein überzeugungsunabhängiges und demnach perspektivenunabhängiges Objekt ansieht, auf das die Verwendung des sprachlichen Ausdrucks festgelegt ist. So ist es möglich, dass die Gehalte (das heißt mit der Identifikation des Bezugsgegenstand inferentiell verbundenen perspektivenrelativen Voraussetzungen und Konsequenzen) in Bezug auf den betreffenden Gegenstand notwendigerweise individuell variieren, aber damit nicht die Kommunikation - das heißt die semantische Koordinierung kognitiver Zustände oder Information - zusammenbricht. Diese direkte referenzbasierte Strategie (und die auf ihrer Grundlage zum Beispiel modelltheoretisch extensionalistisch entfaltete Theorie der logischen Struktur m ö g l i c h e r Extensionsverläufe oder Intension en als Äquivalente für semantische Normen) ist für Brandom wegen des Vermeidungsprinzips inakzeptabel, da sie ihn zur Hinzufügung eines nicht-inferentiellen semantischen Grundbegriffs zwänge. Dennoch dient sie Brandom als Blaupause für seinen semantischen Gegenentwurf.

Mit der perspektivistischen Radikalisierung des Kommunikationsproblems ergibt sich, wie Brandom sagt, aus der Kombination von Inferentialismus und Holismus die These eines systematisch unvermeidlichen "gulf between doxastic perspectives" (Brandom 2010b: 171) zwischen Sprecher und Hörer. Wie erwähnt folgt daraus direkt die Unverwendbarkeit von Kommunikationsmodellen, die eine Perspektivenverschmelzung, Perspektivenübernahme oder geteilte Bedeutungen einer öffentlichen Sprache als telos und Voraussetzung erfolgreicher Kommunikation ansetzen. ${ }^{9}$ Demgegenüber besteht in Brandoms inferentialistischem Holismus die Grundlage jeden Verstehens sprachlicher Äußerungen in den je eigenen doxastischen Repertoires. Daher sind Idiolekte die Verstehen ermöglichenden Bedeutungshorizonte, in die jede Äußerung eingepasst werden muss, um für einen Sprecher als gehaltvoll erkennbar und in ihrem Gehalt bestimmbar zu werden. Die entscheidende A k t i v i tät des Hörers besteht darin, vom Sprecher geäußerte Sätze im e i g e n e n Repertoire wiederkehren zu lassen. Sprecher und Hörer teilen also nicht primär Gehalte, sondern Sätze, Ausdrücke. In der dis k u r s i v e n P r a $\mathrm{x}$ i s werden die behaupteten Sätze dann in unterschiedliche Perspektiven eingebettet und damit interpretiert. ${ }^{13}$ Wissen, was eine Äußerung bedeutet, besteht dann in der Fähigkeit, einen von einem bestimmten Sprecher geäußerten Behauptungssatz so zu interpretieren (das heißt die darin vorkommenden Worte und Strukturen so mit eigenen Ausdrücken zu korrelieren), dass sich daraus im Milieu eigener Festlegungen die Signifikanz ergibt, die sich auch für den Sprecher ergeben würde, wenn er dasselbe doxastische Repertoire hätte wie man selbst. Im Resultat ergibt sich ein Perspektivenkoordinierungsmodell, das kompetent kontoführendes Teilnehmen an der diskursiven Praxis (Verste- 
hen) auf die Kontoführungsfähigkeit zurückführt. ${ }^{11}$ Brandom verwendet hierfür die Metapher der Navigation:

"what is taken to be shared may be different from the points of view of different scorekeepers [...] inferential contents are essentially perspectival [...] What is shared is a capacity to navigate and traverse differences in point of view to specify contents from different points of view" (MIE: 485; Hervorhebung im Original).

Brandoms Strategie zur Lösung des Kommunikationsproblems führt also zunächst zu einer Neudefinition dessen, worin Kommunikationserfolg besteht im Sinne der Informationsextraktion. Sprecherbehauptungen aus deren Herkunftsmilieu in das Milieu eigener als behauptbar angesehener Festlegungen zu übertragen bedeutet die Übernahme des perspektiven u n a b hä n g i g b e i de n verfügba ren (aber auch zunächst semantisch unbestimmten) Äußerungsereignisses durch ein "mapping your noises onto mine, and then [...] extract information from your remark” (2010b: 171). Brandoms Navigationsmodell versteht kommunikativen Erfolg also als das „Extrahieren von Information“ mittels „Interpretation als Korrelation“.

Diese Modellumstellung dient Brandom dazu, das Vermeidungsprinzip durch den konstruktiv-erklärenden Nachvollzug von Referenz effekte $n$ auf referenzfreier Basis von Weiterfassung des Inferentiellen zusammen mit einer ausdruckszentrierten Semantik gegenseitiger Interpretation mittels eines anaphorisch und nicht gegenstandsbezogen vermittelten Korrelation von Sprecher- und Hörer-Äußerungen und den damit verbundenen deontischen Statusveränderungen einzuhalten. Bezüglich des ersteren legt Brandom nahe, dass durch die Erweiterung des Inferenzbegriffs ein Beitrag der Umwelt an der Richtigkeit von Interpretationen mittels der perspektivenüb e rgre ife $n$ de $n$ Einbettung von Sprechern in einer gemeinsamen Umwelt dadurch ins Innere der Praktiken verlegt werden kann, dass die Einschränkung der perspektivenabhängigen Schlusstätigkeit durch Umweltbedingungen inferentiell rekonzipiert wird. Bezüglich des zweiten sieht Brandom die detaillierte Entwicklung der semantischen Normen diskursiv-anaphorischer Anknüpfungen als "offering an account of referential relations to objects in terms ultimately of inferential relations among claims" (AR: 28). Die Kombination von weitem Inferenzbegriff und anaphorisch-substitutioneller Semantik im Navigationsmodell der Kommunikation stellt Brandoms Lösungsvorschlag für das Kommunikations- und Perspektivitätsproblem dar: "adopting the navigation-across-perspectives model of communication can [...] be seen as a way of developing the [...] retreat-to-reference response to the challenge to make communication intelligible within a holistic semantics" (Brandom 2010b: 172).

Im Folgenden möchte ich prüfen, ob der Inferentialismus Brandomscher Prägung tatsächlich eine Lösung des Kommunikationsproblems ohne Inanspruchnahme wesentlich referenzieller Elemente leistet. Zunächst werde ich dabei im nächsten Abschnitt kurz die Suggestion des Nach-Innen-Verlegens von semantischen Beiträgen der Umwelt im weiten Inferenzbegriff als Lösungs- 
ressource für das Kommunikationsproblem kommentieren. Dabei wird sich auch Brandoms eigener Einschätzung nach - herausstellen, dass die bloße Einbettung perzeptuell-praktischer materialer Festlegungen in diskursiv-inferentielle Praktiken entweder ihre Erklärungskraft Referenzinfiltrationen verdankt oder diese systematisch unbestimmt lässt. Um für sozial verbindliche semantische Normen hinzureichen, verweist die Einbettung damit zurück auf die Notwendigkeit zugrundeliegender semantischer Normen gegenseitiger Ausdrucksinterpretation für die Lösung des Kommunikationsproblems. Im Anschluss werde ich deren Entfaltung in Brandoms Inferentialismus auf das Hinreichen zu referenzanalogen semantischen Normierungen untersuchen.

\section{Weiter Inferentialismus und illegitime Referenzinfiltration}

Nur ein Referenzersatz verspricht also die Lösung des Kommunikationsproblems. Unsere inferentiellen Materialien sind, dem weite $n$ Inferenzbegriff nach, bereits mit der Umwelt verbunden, indem unsere informationstragenden (verläs slichen) kognitiven Reaktionen auf dieselbe und Interventionen in sie in Form von doxastischen und praktischen Festlegungen ihre Spur hinterlassen. Es fragt sich daher, ob die Erweiterung ein Korrelat semantischen Weltbezugs in d i r e k t liefert. In der Literatur und in Brandoms Theorieselbstdarstellung wird in Bezug auf die Frage der Einholung referenzieller Grundannahmen häufig die Sellarssche Komponente des Brandomschen Inferentialismus ins Feld geführt, durch die die intellektuellen Folgerungssysteme um die praktisch-perzeptuellen erweitert und damit sozusagen von in ne $n$ mit Weltbezügen aufgeladen werden. Brandom suggeriert nun häufiger, dass sich damit das Bezugsproblem auf die Umwelt sozusagen von selbst löst, da wir es ja nun mit gegenstandsinvolvierten pragmatistischen Intelligenzen und nicht nur mit cartesischen Intellekten zu tun haben. ${ }^{12}$ Dann sei es nur noch ein kleiner Schritt, so die Denkeinladung, von öffentlich durch perzeptuell-praktische Einbettung zugänglichen Objektbezügen zu geteilten semantischen Bezugspunkten überzugehen und damit ein perspektiventranszendierendes Element in ne rhalb der inferentialistischen Ressourcen zu identifizieren. ${ }^{13}$

Diese Suggestion kann aber schon aus einem prinzipiellen Grund nicht hilfreich für die Lösung des Kommunikations- und Perspektivitätsproblem inferentiell definierter Gehalte sein. Die Erweiterung des Inferentialismus auf material proprieties und praktische Eingangs- und Ausgangsbestimmungen macht Sprecher und ihre inferentiell definierten doxastischen und praktischen Repertoires ja zunächst nur zu besser als lediglich sprachlich ausgestatteten praktischen Intelligenzen. Selbst wenn wir es nun mit praktisch involvierten Intelligenzen zu tun haben, wirken sich doch ihre durch Umweltinteraktionen unmittelbar gewonnenen materialen Schlussberechtigungen (MIE: 596) nur auf die jeweiligen individuellen inferentiellen Repertoires aus. Im inferentialistischen Sinne bedeutet ihre Hinzunahme ein Mehr an materialen inferentiellen Ressourcen, aber nicht ein A n d e rs an transpersonaler Verfügbar- 
keit und Signifikanz. In gewissem Sinne verschärft sich das Kommunikationsproblem damit sogar. Da ihre mentalen Zustände nun allesamt informationstragend sind, steht diesen Intelligenzen im Gegensatz zu klassisch empiristischen Ansätzen, in denen wahrnehmungsabhängige materiale proprie ties empirisch und daher nicht bedeutungsbestimmend sind, keine inferentiellen Übergänge mehr als nicht zur Gehaltsbestimmung zähI e n d und bloß empirisch (aber damit auch perspektivenneutral) zur Verfügung. Wenn das Kommunikations- und Perspektivitätsproblem (wie von Brandom zuvor überzeugend dargelegt) eine Folgerung aus der Kombination von Inferentialismus und Bedeutungsholismus ist, dann stellt es sich auch und gerade für weit-inferentialistisch ausgestattete Intelligenzen. Daher kann der Hinweis auf weite Inferentialität im Sinne der incorporation of material (MIE: 623) höchstens Teil des Problems sein, aber nicht selbst die Lösung. ${ }^{14}$

Dass Brandom sich dennoch einen Lösungsbeitrag von der Einführung des weiten Inferenzbegriffs erhofft, ohne diesen auf die kausale Bestimmung von Dispositionen zu reduzieren, erkennt man daran, dass er auf einen Einwand von Fodor/Lepore (2010) gleicher Stoßrichtung den w e it e n Inferenzbegriff ins Feld führt, um die Konsequenz aus dem perspektivistischen Bedeutungsholismus als $z$ u extrem zu entkräften, dass "we share nothing". Sein Argument lautet:

"we do share the words, at least in the sense of noise [and] mapping the noises made by the interpretive target onto the noises made by the interpreter [...] recall that inferential role in the broad sense includes the inferential connections between circumstances of appropriate application and appropriate consequences of application quite generally - even when circumstances or consequences of application themselves are noninferential" (Brandom 2010b: 170f.) $)^{15}$

Doch wenn wir die zuvor genannte Perspektivenrelativität des Gehalts ernstnehmen, kann es nicht ohne Weiteres überzeugen, dass

"these language entry and language exit moves, no less than the language-language ones, also give us something important in common [...] I do not see why the structures so described do not underwrite a perfectly intelligible notion of partially shared [...] inferential roles" (Brandom 2010b: 171).

Das Problem besteht in der Behauptung der Gemeinsamkeit und Geteiltheit (im Unterschied zu der von keiner Seite angezweifelten Öffentlichkeit) der Umstände. Da es sich bei den betreffenden Informationen um materiale Substitutionsschlüsse und ähnliche Zusatzannahmen handelt, ist nicht klar, weshalb die Öffentlichkeit und der non-inferentielle (das heißt nicht inferenzabhängige) Charakter der Umstände als solcher zur beiderseitig verpflichtenden Veränderung deontischer Status oder auch nur Einstellungen führen sollte. Dadurch wird ja die Information selbst nicht nicht-inferentiell. Schließlich ordnet ja, wie gesagt, Brandoms Perspektivismus zufolge jeder die entspre- 
chenden Informationen in sein eigenes Hintergrundrepertoire ein. Auch der Hinweis auf die Öffentlichkeit der Umstände diskursiver Praxis weist also keine im Sinne des Inferentialismus nicht-perspektivische gehaltsbestimmende Ressource auf, solange der Zusammenhang mit normativ erforderlichen Statusveränderungen nicht klar ist.

Brandom bedient sich hier an den entscheidenden Stellen immer wieder natürlicher, von inm als inferentiell deklarierter (aber eben nicht erklärter) Übergänge von kausalen Beschränkungen diskursiver Praxis zu normativen Bestimmungen semantischer Gehalte. Diese werden dann als adäquate inferentialistische Korrelate für referenzielle Elemente in der Gehaltsspezifikation weiterverwendet und für den Aufweis herangezogen, dass eine so e rwe it e r te inferentialistische Position die inferentiellen Effekte erfolgreicher demonstrativer Bezugnahme besser erklären könne als die herkömmliche referenzielle Interpretation.

Letztere sieht sich ja dem Problem gegenüber, wie ein nicht als propositionaler Gehalt erscheinender außersprachlicher Gegenstand in das Spiel und Gegenspiel von Gründen eingreifen und dies so beschränken können soll. Die Erwe i terung soll dabei helfen, den so benannten My thos des Gegeb e n e n durch die Identifikation von p ri mä $r$ propositionalen und erst sekundär (durch propositional und kommunikativ erfolgende Identifikationsleistungen) referenziell zu reinterpretierenden Informationsaufnahmen aus wahrnehmender Interaktion mit Umweltumständen zu entkräften, o h n e bei den primären Bezügen referenzielle Annahmen einzuschmuggeln. Repräsentativ für diese Argumentationslinie ist MIE, Kapitel 5.V.4., wo Brandom bei der Erläuterung des weiten Inferenzbegriffs gleitend von der Existenz von e m pirical and practical constraints für jeden Kontoführer über proprieties materialer Inferenzen (die in diesem Zusammenhang darin bestehen, dass man kontingent nicht zu allem berechtigt ist, worauf man sich festlegt, weil einige Festlegungen unmittelbar umweltlich bedingt sind) zu co n ceptual proprieties übergeht. Daraus schließt Brandom, dass es eine "causal contribution to the norms" gibt, die Sprachgemeinschaftsmitglieder dazu instand setzt,

"to have undertaken commitments by using [...] words that outrun their capacity to recognize these commitments, [so that] I can find myself with incompatible commitments [...]. The possibility of incompatible commitments arising from the cycle of perception, inference, action, and perception reflects the way the normative structure [...] incorporates elements of the causal order" (MIE: 331f.).

In ähnlicher Weise beschreibt Brandom die Einbeziehung der kausalen Ordnung als Domestizierung des Normativen: "Normative statuses are domesticated by being understood in terms of normative attitudes, which are in the causal order" (MIE: 626; zweite Hervorhebung A.M.).

Wie ist es aber genauer verständlich zu machen, dass einem im Verlauf von Wahrnehmung, Schluss, Handlung und Wahrnehmung eines Resultats der deontische Status zuwächst, miteinander inkompatible Festlegungen zu besit- 
zen? Brandom zufolge hängen die für eine Sprecherin tat sä chlich geltenden Festlegungen, Berechtigungen und Inkompatibilitäten nicht nur davon ab, welche Festlegungen, Berechtigungen und Inkompatibilitäten sie selbst unternimmt, beansprucht oder feststellt, sondern auch davon, welche davon von anderen Sprecherinnen anerkannt, übernommen oder bestritten werden können. Um diese Anerkennungen, Übernahmen und Anfechtungen (das heißt, die für die Produktion des Sprecherstatus verantwortlichen Einstellungen) vornehmen zu können, müssen die fraglichen anderen jedoch zunächst identifizieren können, um welche Festlegungen (und so weiter) es sich bei den Behauptungen der Sprecherin gehandelt hat und somit Normen dafür unterstellen, wie das Sprachverhalten fü $r$ a lle (sie selbst und die Sprecherin) so zu verstehen ist, dass bestimmte geäußerte Worte bestimmte Festlegungen (und so weiter.) ausdrücken. Demnach baute aber nun die soziale Wechselwirkung normativer Einstellungen zur Produktion transpersonal identischer normativer Status (wie Berechtigung) auf stillschweigend mitlaufende semantische Normen auf. In dazu analoger Weise kann sich ein einzelner Sprecher nur dann miteinander inkompatiblen Festlegungen gegenübersehen, wenn diese so bestimm t sind, dass der Ausdruck der einen Festlegung als Behauptung der Berechtigung zur Behauptung der anderen wide rsp richt under deswegen nur eine der beiden unternimmt. Das ist jedoch die entgegenge setzte Erklärungsrichtung, wie die von Brandom intendierte.

Das die betreffende Stelle elaborierende Beispiel erlaubt es, dies zu verdeutlichen:

"So if I perceive a liquid as tasting sour, infer that it is an acid, infer further that it will therefore turn litmus paper red, and, intending to match a red pigment sample, accordingly dip litmus paper in the liquid, I may nonetheless subsequently acquire perceptually commitment to the result being a blue [...] piece of paper, and hence an acknowledgment of my practical failure. [...] As a result, empirical and practical constraints get built into what commitments (including inferential commitments) one can sustain entitlement to" (MIE: 332f.; Hervorhebung A.M.). ${ }^{16}$

Es ist nur dann der Fall, dass ich das unerwartete Resultat des Tests als „failure" identifizieren sollte statt als Zuwachs an bedeutungsbestimmender Information, wenn fürmich unabhängig vom Testresultat bereits festliegt, dass das „acid“ nur dann korrekt angewendet ist, wenn es Lackmuspapier rot färbt (das heißt die Referenz von acid das ist, wie auch immer es korrekt beschreibbar sein möge, was Lackmuspapier rot und nicht anders färbt) und ich damit den Status der Berechtigung zu dieser Fests etzung fixiere (MIE: Kap.1.VI.2). ${ }^{17}$ Das ist allerdings eine semantische Festlegung, die ausschließt, dass acid der Ausdruck für all das ist, was Lackmuspapier manchmal rot und manchmal blau färbt (was acid in der öffentlichen Sprache oder bei Experten auch verlangen mag). Es ist natürlich nicht inferentiell verboten, die Blaufärbungsbeobachtung als Korrektur der Bedeutung von acid anzusetzen. Es ist aber se man $\mathrm{t}$ i s $\mathrm{ch}$ verboten, wenn alles mit acid korrekt Beschreibbare das zu sein hat, was Lackmuspapier rot 
und nicht anders färbt (das heißt dieser Kausalzusammenhang als $\mathrm{S}$ t a n dardprozess für die Weiterverwendung von acid fixiert ist). Nur dan $n$ kann ich folger $n$, dass eine Verpflichtung zum Einstellungswandel von "undertaking the commitment that what tastes sour is an acid" zu "acknowledgment of failure" besteht. Das heißt der Status, miteinander unvereinbare Festlegungen zu bemerken und im Lichte unerwarteter Wahrnehmungen die Einstellung zu einigen meiner Festlegungen zu korrigieren, hängt von dem in f e re n z i m m u n festliegenden Status der Berechtigung zur Fixierung des semantischen Werts von acid mittels eines Standardprozesses ab, der die intuitiv bedeutungsverändernde Inferenz unterbindet. ${ }^{18}$ Das gilt unabhängig von der Tatsache, dass Brandom hier ja einen Fall praktischer Lernprozesse betrachtet, in die scheinbar keine (bewussten) Repräsentationen eingehen, denn es ist für die Darstellung der Handlungsabfolge hier wesentlich, dass ich darauf schließe, dass es sich um eine Säure handelt, was sicherlich k e in lediglich praktischer, sondern ein wesentlich situationserfas s e n d-d a r s t e I l e n d e r und erst dadurch hypothesengenerierender kognitiver Schritt ist. ${ }^{19}$ Der inferentielle Lernerfolg hängt hier von der Vor a u s setzung von de facto referenziellen (bezugsfixierenden) Annahmen ab, nicht umgekehrt die B e re chtigung zu konstruktiv erzeugten referenzanalogen (aber fundamental inferentialistischen) Interpretationshypothesen von referenzfreien (freistehenden) Folgerungsketten. ${ }^{20}$

Ohne festliegende Interpretation en der words (oder Anerkennung von unmittelbar aus der feststellenden Autorisierung nichtperspektivischer Umweltzustände durch erstpersonale objektabhängige Behauptungen ${ }^{21}$ ) könnte die fragliche "incorporation of the causal order" und der damit einhergehenden unerwarteten Wahrnehmungen - deraus dem Holismus resultierenden Unterbestimmtheit zufolge - immer nur die disjunktive Signifika n z erreichen, e n t w e der eine Veränderung der Bedeutung der Worte (ihre Anwendung auf Dinge, die der alten Regularität zufolge noch nicht dazuzählten) o de r eine Inkompatibilität erzeugen. Nur dann, wenn die Beiträge der kausalen Ordnung le gitimiert als Beiträge zur propriety von Schlüssen (zum Beispiel auf Inkompatibilität) aufgefasst werden, kann von ihnen überhaupt so etwas wie ein Zwang ausgehen, der den eigenen Festlegungen und Berechtigungen gegenüber autoritativ ist. ${ }^{22}$ Und das ist nur dann der Fall, wenn festliegt, welche Erfahrungen zu welchen Sprachgebrauchsmustern in Gegensatz treten und somit, we l c he Gebrauchsmuster se man tisch normativ und erst damit inferentiell relevant sind. Die Möglichkeit des "outrun their capacity to recognize" kommt also nur e n tw e de $r$ unter Voraussetzung festliegender Bezugnahmeregeln oder nicht $\mathrm{z}$ wingend zustande. ${ }^{23}$ Da das Kommunikationsproblem wegen des Holismus nur auf referenzanaloge Weise gelöst werden kann und als gemeinsam identifizierbare Referenz durch kausale Einbettung jedes Einzelnen inferentiell auf diese Reagierenden nicht hinreichend bestimmbar ist, stellt der Hinweis auf die Erweiterung des Inferenzbegriffs also höchstens eine Erweiterung des Kommunikations problems um die Frage dar, wie die Festlegung semantischer 
Regeln/Normen durch referenzanaloge aber referenzfreie Mittel erfolgen kann, aber nicht eine mögliche Lösung desselben. ${ }^{24}$

Die vorgeblich erfolgreiche referenzanaloge aber referenzfreie Erklärung von Gehaltskontinuität ist somit in den Beispielen und der Berufung auf den weiten Inferenzbegriff als e rfolg t vorausge se tz t und nicht dort geleistet. Brandoms Beispiele kognitiver Effekte von wahrnehmungsbasierten Selbstkorrekturen sind $\mathrm{m}$ it referenziellen Annahmen völlig einleuchtend und erhellend. Im Anschluss an Lycan (1986: 258) könnte man von „Referenzinfiltrierungen“ in Brandoms Darstellung sprechen, ${ }^{25}$ die sich als Nebeneffekte der kohärenten Beschreibung bestimmter Beispiele fast unwillkürlich einstellen. Dem Vermeidungsprinzip zufolge sind sie jedoch unzulässig für die Lösung des Kommunikationsproblems und des Perspektivenrelativitätsproblems sprachlichen Gehalts. Fairerweise ist zu sagen, dass Brandom selbst nicht explizit behauptet, mit diesen Hinzufügungen allein das Kommunikationsproblem zu lösen; im Gegenteil ordnet er Erwägungen des umweltlich Geteilten Handlungshintergrunds dem "so far sub-semantic level" (Brandom 2010b: 171) zu. Brandoms Diskussion beruht in diesen Zusammenhängen also auf der Vo r w e g $\mathrm{n}$ a $\mathrm{h} \mathrm{m}$ e eines diese Unterscheidungen und Bestimmungen leistenden referenzfreien aber referenzanalogen Mechanismus. Diesem wende ich mich nun zu.

\section{Anapher als Lösungsvorschlag}

Brandoms Navigationsmodell zufolge verlangt die Lösung des Kommunikationsproblems eine Erklärung der "capacity [...] of the audience to pick up a speaker's tokening [to] [...] connect it to their own substitution-inferential commitments is part of what makes it possible [...] (to extract) information from it"(MIE: 475). Wie gerade gesehen, reicht die We i t e r f a s s ung des inferentiellen Materials nicht für diese Aufgabe hin, da sie den Beitrag der informationshaltigen Umwelt zu Veränderungen der kognitiv-inferentiellen Repertoires von Sprechern unbestimmt und perspektivenrelativ belässt. Für eine solche Bestimmung und das Erben so bestimmter (und nicht anderer) Information von Ausdrucksäußerungen anderer bedarf es semantischer Normen die von Sprechern geäußerte Ausdrücke spezifisch sensitiv auf Veränderungen der materialen Informationslage machen. Deren Integration in existierende inferentielle Milieus muss für jeden diesen Ausdruck übernehmenden Hörer in bestimmter (und nicht anderer) Weise signifikant sein. Solche semantischen Mechanismen und Normen erläutert Brandom in seiner substitutionell-anaphorischen Semantik in Kapitel 5-7 von MIE. Ohne näher auf die andernorts ausführlich dargestellten Details der teils genialen Kunstgriffe in Brandoms Durchführung des Programms einer inferentialistischen Semantik eingehen zu wollen, empfiehlt es sich an dieser Stelle, seine inferentialistische Konzeption stehender - und in diesem Sinne verbind licher - lexikalischer Bedeutungen kurz einzuführen, um Verwechslungen mit anaphorisch g e erb te n Gehalten aus dem Weg zu gehen. 
Die stehende Bedeutung wiederkehrender Satzkomponenten im Milieu der Festlegungen eines kompetenten Sprechers erklärt Brandom im Sinne einer Erweiterung der inferentialistischen Analyse von der Ebene der (direkt als Prämissen und Konklusionen verfügbaren und damit signifikanztragend e n ) Sätze auf die der Satzkomponenten wie folgt: Die Bedeutung eines subsententiellen, an der Subjektstelle einer einfachen Behauptung auftretenden Ausdrucks besteht in dessen indirekt inferentieller Rolle, und diese wiederum ergibt sich aus den festlegungserhaltenden Substitutionsschlüssen, in die er eingeht. In welche Substitutionsschlüsse ein gegebener Ausdruck eingeht, hängt wiederum von den „einfachen materialen substitutionsinferentiellen Festlegungen“ (SMSICs; MIE: 373) des den Ausdruck verwendenden Sprechers ab. Diese einfachen materialen Festlegungen bestimmen in ihrer Gesamtheit die Gesamtbedeutung des in ihnen vorkommenden Ausdrucks mittels dessen Rolle in einem System von Substitutionsschlüssen. Diese Substitutionsschlüsse lassen sich beispielsweise mit Bezug auf die Bedeutung von Goethe dahingehend charakterisieren (Wanderer 2008: 131), dass der Schluss von (1) Goethe X auf (2) Der Autor von Iphigenie auf Tauris $X$ material gut ist. Die Gesamtsignifikanz des Ausdrucks Goethe für einen Sprecher besteht demnach in der Gesamtheit der inferentiellen und s u b s t it u t i o n e Il e n Festlegungen, an denen er Teil hat. ${ }^{26}$ Dass die Hinzufügung substitutioneller Festlegungen zum inferentiellen Repertoire das Perspektivismusund Kommunikationsproblem nicht lösen kann, sollte klar sein, denn durch die Abhängigkeit der Richtigkeit der Ersetzungen von einfachen materialen Festlegungen sind diese ja Teil der für den jeweiligen individuellen Sprecher zu bestimmenden Signifikanz und somit Teil der perspektivencharakterisierenden Festlegungen.

Um von diesen perspektivenrelativen Ressourcen für die Extraktion von Information aus Sprecheräußerungen zu perspektivenneutralen Bezugspunkten für Sprecher und Hörer und damit der Herstellung von kommunikativen Verknüpfungen zu gelangen, bedarf es also eines weiteren Analyseschrittes, der unterschiedliche Substitutionsrepertoires miteinander durch so etwas wie Brückenprinzipien verbindet. Dabei wird nun wichtig, dass es sich bei Kommunikation um eine diskursive P raxis der gegenseitigen Äußerungsinterpretation und damit ein Verhältnis von Sprachverwe ndern handelt. Schon die substitutionstheoretische Erklärung der Signifikanz subsententieller Ausdrücke greift ja aus g e b ra u c h s th e o re tischer Sicht auf eine weitere Struktur zurück, wie an einer Analyse der Effekte auf ein System von Festlegungen ersichtlich wird. Substitutionsschlüsse dieser Struktur sind genau dann festlegungserhaltend, wenn sie dazu berechtigen, einen Ausdruck in einem (eine bestimmte Festlegung ausdrückenden) Behauptungssatz durch einen mit ihm in einem einfachen materialen Substitutionsschluss assoziierten Ausdruck zu ersetzen.

Beispielsweise ist die Festlegung darauf, dass Goethe hessisch sprach d i e s e l b e wie die, dass der Autor von Iphigenie auf Tauris hessisch sprach, das heißt sie folgt aus denselben Voraussetzungen und erlaubt dieselben Folgerungen im Kontext der Gesamtheit von Substitutionsschlüssen, auf die ein 
gegebener Sprecher festgelegt ist, da man diese Ersetzung ja jederzeit wieder rückgängig machen kann, indem man den Ausdruck Goethe wieder für der Autor von Iphigenie auf Tauris ersetzt. Daraus wird jedoch auch klar, dass Substitutionsschlüsse auf wiederkehrende Ausdruckstypen bezogen definiert sind. Nimmt man es gebrauchstheoretisch genau, dann besteht die semantische Signifikanz von substitutionellen Netzwerken darin, dass jede Äußerung des Ausdruckstyps Goethe in einem indikativen Behauptungssatz ohne indirekte Rede durch ein token /Goethe/durch eine Äußerung des Ausdruckstyps der Autor von Iphigenie auf Tauris in demselben Satz durch ein token /der Autor von Iphingenie auf Tauris/ersetzt werden kann. Somit verlangt die Anwendung von Substitutionsregeln auf die Satzverortungen (occurrences) bestimmter Ausdrücke in Äußerungskontexten die Annahme, dass die in diesen Äußerungen enthaltenen tokens Rekurrenzen (oder semantisch in e rte Äußerungswiederholungen) darstellen. ${ }^{27}$ Diese semantisch inerten (aber potentiell, im homophonen Fall der Rekurrenz, ausdruckstypisierenden) Äußerungswiederholungsstrukturen entnimmt Brandom dem anaphorischen Modell.

Die Grundlage für Brandoms Modell bildet die sogenannte Diskursanapher oder Faulheits a napher ${ }^{28}$, in der man die Wiederholung langer Ausdrücke für den Bezug auf Gegenstände durch pronominale Ausdrücke vermeidet, wie beispielsweise in dem Satz Der Autor von Iphigenie auf Tauris sprach hessisch, weil er aus Frankfurt am Main war. Das Pronomen e $r$ stellt Goethe nicht dar und fügt keinerlei beschreibende Information hinzu (ist also in diesem Sinne „semantisch inert“ (MIE: 303). Es erfüllt eine F u n k ti o n, nämlich die eines Platzhalters in der Satzstruktur, der den Gehalt eines vorhergehenden Äußerungsausdrucks zum Satz beiträgt, von dem er a b h ä n g t. Solche Anaphern sind Mittel des zurückverweisenden Gehaltserhalts. ${ }^{29}$ We Ich e n Gehalt beispielsweise er in einem Satz hat, hängt dabei nicht wesentlich davon ab, was er bedeutet, sondern nur von dem (von Sprechern frei wählbaren und im Beispiel als Der Autor von Iphigenie auf Tauris festgelegten) Ausdruck, auf den er zurückzuverweisen hat. ${ }^{30}$ Anaphorische Rückverweise sind jedoch nicht von der Präsenz von Pronominalausdrücken abhängig - diese zeigen lediglich, dass es für die erfolgreiche Erfüllung der anaphorischen Funktion (bis auf syntaktische bzw. grammatische Bindungselemente wie Genus und Numerus) keiner eigener deskriptiver semantischer Eigenschaften des zurückverweisenden Ausdrucks bedarf. Anaphorische Verbindungen sind im Gegensatz zu Substitutionsschlüssen auf Ausdrucksereignisse oder tokens bezogen definiert, weswegen jede andere identifizierende Bezugnahme auf diese tokens ein funktionales Äquivalent zum Pronomen liefert, so lange sie die Verbindung zum selben Antezedens aufrechterhält. Anaphorische Verbindungen bieten damit die gesuchten Rekurrenzstrukturen, derer sich Substitutionsschlüsse implizit bedienen. Das Resultat anaphorischer Rückverweise ist eine Ve rke t t u ng von Ausdrücken beliebigen lexikalischen Typs, solange der verwendete Ausdruck im Anschlusssatz es gram matis ch erlaubt, auf das vorhergehende Kettenglied im Antezedenzsatz Bezug zu nehmen. Jede anaphorische Fortsetzung der Kette wiede- 
rum ist dadurch definiert, dass sie Gehalt lediglich von vorherigen Gliedern übernimmt. Somit haben alle Glieder einer gegebenen anaphorischen Kette diese lbe Be deutung, die darin enthaltenen Ausdrucksereignisse bilden also eine semantische Äquivalenzklasse.

Eine bestehende anaphorische Kette lässt sich hinsichtlich des Gehalts der in ihr vorkommenden token als „Intersubstituierbarkeitsklasse“ (das heißt das Produkt aus „Rekurrenzklasse“ (MIE: 457) und Substitutionsberechtigung) auffassen und damit p ra g m a t i s c h als Klasse von Ausdrucksvorkommnissen, von denen jeder Sprecher gleichermaßen weiß, dass sie füreinander ersetzt werden können, ${ }^{31}$ und somit, dass es für die Berechtigung dieser Ersetzungen keine Rolle spielt, wer und unter welchen Substituierbarkeitsfestlegungshintergründen ein Vorkommnis geäußert hat. Es handelt sich hierbei um einen $\mathrm{E} f \mathrm{fe} \mathrm{kt}$ der ( s t i p u li e r t e n ) Verkettung, den Brandom als entscheidend für die metasemantische Identifikation der semantischen $\mathrm{N}$ atur des Phänomens anaphorischer Verknüpfungen betrachtet. Diesem Effekt zufolge, dass es sich nämlich bei den miteinander verketteten tokens um eine Intersubstitu tionsklasse handelt und Intersubstitution von anaphorisch verketteten tokens eine Art von Substitution ist, und Substitution eine in ferenti e lle Beziehung ist, nimmt Brandom die These der inferentiellen Natur der Semantik anaphorischer Verkettungen, die ich als IN abkürze, als erwiesen an:

(IN) Anaphorische M e chan is me $\mathrm{n}$ selbst sind rein inferentielle (und alle anderen semantischen Beziehungen fundierende) Phänomene. ${ }^{32}$

Drei bislang eher beiläufige Aspekte von Brandoms Modellierung anaphorischer Ketten sind wichtig dafür zu verstehen, inwiefern ihm zufolge anaphorische Ketten (die ja im Sinne der deontischen Status in der Kommunikation Substitutionsberechtigungen darstellen, die ein sie stipulierender Sprecher als Festlegung erwerben kann) anders als perspektivische Substitutionsfestlegungen nicht perspektivisch, sondern perspektivenneutral verfügbar und alle Sprecher unter eine gemeinsame semantische Norm bringend anzusehen sind.

(A1) Erstens sind die Ketten nämlich als auf raumzeitlich verortete Ausdrucksvorkommnisse definierte Verknüpfungen öffentlich zugänglich (MIE: 311). (A2) Zweitens werden erst durch anaphorische Strukturen bestimmte Ausdrucksklassen als Kandidaten für substitutioneller Strukturen erkennbar (und in diesem Sinne gehen öffentlich zugängliche Verknüpfungsereignisse semantisch perspektivencharkaterisierenden ,substitutionellen' Identifikationsverknüpfungen voraus). ${ }^{33}(A 3)$ Drittens sind sie als durch Sprecher und Hörer im Vollzug diskursiver Fortsetzung stipulierte Verweise sozusagen direkte semantische Normierungen von Ausdrucksklassen miteinander verketteter Vorkommnisse als gehaltgleich, die mittels der Fähigkeit zu anaphorisch akzeptablen Fortsetzungen eines Dialogs zum Ausdruck kommen und, sobald so ausgedrückt, von Brandom als für beide Seiten gleichermaßen verpflichtend postuliert werden ${ }^{34}$ : 
"Anaphoric chains running through bits of discourse [...] are normative features attributed to the discourse by deontic scorekeepers [...] of the obligation that the significance assigned to [...] one part of the discourse answer in systematic ways to the significance assigned to [...] another" (MIE: 460). ${ }^{35}$

Der für Brandoms referenzanaloge aber (angeblich) referenzfreie Erklärung der Kommunikation entscheidende Witz an anaphorischen Abhängigkeiten ist, dass anaphorische Abhängigkeit (laut (A1)) als Bezug auf das Verwendungsereignis eines Antezedenzausdrucks im Gegensatz zu der mit dem Ausdruck von einem Sprecher verbundenen Signifikanz definiert ist. Anaphorische Ketten sind in diesem Sinne ne u tral (laut (A2) sogar vorgängig) gegenüber den perspektivisch unterschiedlichen Interpretationen der Ausdrucksereignisse und (da nachfolgende Kettenglieder ja - laut (A3) - a b hä ng ig von vorherigen und damit nicht eigenständig semantisch bewertet werden können) dennoch s tipula ti v als korrekt gehaltserhaltende Verkettungen erkennbar:

"Use of an anaphoric proform implicitly stipulates coreference with the anaphoric antecedent [...] Thus differences in the substitutional commitments [...] according to speakers and audience can be bracketed and a common topic of conversation secured by using a tokening that is anaphorically dependent. [...] Interpersonal anaphora achieves just the effect that matters for securing communication in the face of differences in collateral commitments" (MIE: 486).

Mit den in (A1)-(A3) aufgeführten semantischen und pragmatischen Eigenschaften weist Brandom anaphorische Ketten als (a) Strukturen der „Vererbung" von Gehalt (MIE: 283) und (b) zureichende Bedingung zur Sicherung eines gemeinsamen Gesprächsinhalts (common topic) aus. Die in der anaphorischen Anknüpfung an in der diskursiven Praxis zeitlich vorangehende Äußerungen stellt demnach die f u n d a m e n t a le semantische Fähigkeit von Sprechern dar:

"it permits each interlocutor to produce utterances employing tokenings that have been stipulated to be recurrences of arbitrary tokenings by others. [...] such recurrences provide the basic points of contact between different repertoires of commitments" (MIE: 458).

Brandom hatte ja zuvor den grundsätzlichen Lösungsansatz mit aus inferentialistischen Prämissen folgenden Gründen im referenziellen Bereich angesiedelt, dessen Annahmen aber nur unter inferentialistischer Korrektur in seine Semantik eingegliedert werden können (MIE: 477ff.). Die Sprechern und Hörern universal verfügbare Fähigkeit zur anaphorischen Bezugnahme auf vorhergehende Redebeiträge ist die von Brandom gesuchte referenzanaloge Struktur, die eine Erklärung der Kommunikation über perspektivisch verfasste, inferentiell signifikanzbestimmende individuelle doxastische Repertoires hinweg leisten soll: "Anaphora is a mechanism that permits undertaking and attributing 
commitments concerning objects that one need not be able to specify" (MIE: 487).

Wie sieht nun die erhoffte Erklärung der Kommunikation aus? Die Grundstruktur scheint in drei Schritten zu verlaufen: (E1) Weil und nur insoweit als dialogkompetente Sprecher den Mechanismus der anaphorischen Anknüpfung als grammatische Kompetenz beziehungsweise (einstellungsunabhängig verfügbaren) Mechanismus ${ }^{36}$ diskursiven Rückverweises beliebiger Gehalte beherrschen, sind sie dazu in der Lage, unter Absehung von der perspektivenabhängigen Signifikanz der Wortw a h I des Sprechers eine tatsächliche Sprecheräußerung in das eigene Vokabular abzubilden und mit Hilfe desselben auszudrücken, o h ne dab e i unterschiedliche semantische Werte annehmen zu müssen, da sie ihre eigene Interpretation beziehungsweise Wiedergabe der Äußerung durch eine praktisch für den Vorredner erkennbare anaphorische Anknüpfung als anaphorische Anknüpfung festsetzen können.

(E2) Weil und nur insofern als jeder mit der grammatischen Struktur verträgliche Ausdruck für solche Anknüpfungen lizensiert ist und dies Sprecher und Hörer bekannt ist, und weil und insofern als jeder solche Ausdruck durch substitutionelle Verknüfungen in das jeweils eigene inferentielle Repertoire eingebettet ist, handelt es sich bei anaphorisch verknüpften Ausdrücken aus beiden voneinander unabhängigen Perspektiven um für sie selbst als gehaltvoll erkennbare Ausdrücke.

(E3) Weil und nur insofern als dies der Fall ist, ist der Semantiktheoretiker dazu berechtigt, anaphorische Ketten als Gehalte vermittelnde und (wegen der Sprechern und Hörern als stipuliert gemeinsamen semantischen Substitutionserlaubnissen in anaphorischen Ketten) als Äquivalenzklassen gehaltgleicher Ausdrücke theoretisch aufzufassen. Da nun alle am Dialog beteiligten Sprecher als berechtigterweise von der Gehalthaftigkeit aller Ausdrucksvorkommnisse ausgehend darstellbar sind und sämtliche Ausdrucksvorkommnisse als gehaltvermittelnd und, per Stipulation durch semantische Normen als gehaltgleich identifizierbar sind, handelt es sich auch bei Abweichungen doxastischer Horizonte um Fälle erfolgreichen Kommunizierens im Sinne geteilter (gegenseitig zuschreibbarer und identifizierbarer) Gehalte.

Unter Annahme des Erklärungserfolgs des in (E1)-(E3) vorgeschlagenen und lediglich auf $(A 1)-(A 3)$ rekurrierenden Modells folgt dann aufgrund der metasemantischen These der inferentiellen Natur der Anapher (IN) weiter, dass die Lösung des Kommunikationsproblems a uf rein inferentialis tischer Grundlage steht (das heißt keine repräsentationalisti$s c h$ e $n$ beziehungsweise referenzinvolvierenden Zusatzprämissen benötigt). Die dreischichtige inferentiell-substitutionell-anaphorische Semantik bietet demnach zureichende Bedingungen für die in diskursiven Praktiken über doxastische Gräben hinweg erfolgenden gegenseitigen Gehaltszuschreibungen (MIE: 484ff.).

Wenn die mittels $(A 1)-(A 3)$ in $(E 1)-(E 3)$ vorgenommene Modellierung die Lösung des Kommunikationsproblems in Brandoms Theorie adäquat zusammenfasst, dann bedarf es mehrerer erläuternder Kommentare hinsichtlich der 
damit erreichten Erklärungsebene. Zunächst ist klar, dass es sich bei dieser Erklärung um eine metasemantische Erklärung handelt, die den Begriff anaphorischer Verknüpfung als dialogkonstitutiven Begriff identifiziert und damit von anderen Kandidaten im referenziellen Repertoire unterscheidet wie zum Beispiel Deixis, bestimmte Beschreibungen, Eigennamen oder Extension. Hierher gehört auch Brandoms aufwendiger Nachweis in Kapitel 7 von MIE, dass die Signifikanz deiktischer Bezugnahmen ohne einen anaphorisch kodierten Hintergrund der Weiterverwendung der in deiktischen Bezugnahmen verwendeten tokens keine kommunizierbaren oder kognitiv verwertbaren (das heißt informationsverändernden) Konsequenzen haben kann. Zweitens wird auch klar, dass die von Brandom ausführlich analysierten, epistemisch-dialogischen Zuschreibungs- und Anerkenntnismechanismen in Kapitel 8 von MIE, die ihrerseits grundlegend für die adäquate Zuweisung und Nachverfolgung deontischer Status im Begründungs- und Behauptungsaustausch sind, n u r a ufg r u nd der Annahme anaphorischer Fähigkeiten so funktionieren können, wie Brandom sie beschreibt. ${ }^{37}$ Denn die in der Abstandnahme von in Sprecheräußerungen enthaltenen und zum Ausdruck kommenden Urteile und Festlegungen im Rahmen von de re-Zuschreibungen, in denen der Hörer die Sprecherausdrücke durch eigene ersetzt, um denselben Gehalt im Milieu eigener Festlegungen konstruktiv wiederzugeben, beruhen offensichtlich auf der Annahme einer zu dieser Ersetzung (diesem substitutionsinferentiellen Schluss) berechtigenden und von beiden anerkannten semantischen Norm, eben die anaphorischer (statt ausdruckserhaltender) Ersetzungen.

Drittens beruht die auf diesen perspektivenverschränkenden Zuschreibungspraktiken aufbauende Möglichkeit der Revision des berechtigten deontischen Status einer eigenen doxastischen Festlegung durch die erfolgreiche Kritik mittels anderer Festlegungen und damit Brandoms Erklärung des Sinns von Objektivität ebenfalls auf der Verfügung über allseits anerkannte anaphorische Mechanismen der Anknüpfung an Festlegungen ausdrückende Diskussionsbeiträge. Angesichts dieser Begründungsaufgaben ist auf jeden Fall deutlich, dass anaphorischen Mechanismen im Rahmen von Brandoms größerformatigen Vorhaben genau die Rolle zukommt, die der Bezugnahme auf Gegenstände einer allen Sprechern gemeinsamen Umwelt in referenzbasierten Ansätzen zukommt, nämlich die, Sprechern gemeinsame Diskussionsgegenstände über Überzeugungsdifferenzen hinweg genauso zu liefern wie dieselben als das Maß dessen anzusetzen, was Sprecher über Kritik hinweg gerechtfertigt als korrekte Überzeugungen annehmen dürfen.

\section{Anapher als unzureichendes semantisches Korrelat der Referenz}

Der Erfolg der gerade vorgeschlagenen Erklärung auf metasemantischer Ebene soll hier genauso wenig in Frage gestellt werden wie die Korrektheit der pragmatischen Analyse von einstellungsüberbrückenden Gehaltszuschreibungen im Sinne der Fähigkeit zur reflexiven Inanspruchnahme der Differenz zwischen der Berechtigung zu (perspektivenabhängigen) Substitutionsfestlegungen und 
der Berechtigung zu (frei stipulierbaren und lediglich als adäquate Anschlüsse) anaphorisch verpflichtenden Gesprächsfortsetzungen. Doch der Nachweis der fundamentalen Rolle anaphorischer Anknüpfungen als eines grundlegenden pragmatischen Repertoires zur Dialogfortsetzung impliziert nicht ohne Weiteres, dass diese Mechanismen selbst referenzfrei zu Sprechern als semantisch bestimmt (und somit erstens als in bestimmter Weise mit perspektivenabhängigen epistemischen Festlegungen interagierende als so bestimmt gemeinsamen) verfügbaren Gehalten hinreichen. Diese für das Funktionieren der Erklärung gemachte Voraussetzung ist nicht metasemantisch, sondern nur im Rahmen einer semantischen Theorie erhältlich, die charakterisiert, wie unterschiedliche anaphorische Ketten ihre unterschiedlichen Gehalte erhalten und somit, falls ein Sprecher von einem Hörer mittels einer unterscheidbaren Kette interpretiert wird, als inkorrekte Gehaltsidentifikation erkennbar sind. Schließlich ist es ja äußerst unplausibel, Sprechern zuzumuten, jede beliebige Stipulation von Gehaltsgleich heit als gleich akzeptabel in einer Diskussionssituation anzusehen, da sie dann ke in e rle i G rund haben anzunehmen, dass die Hörerbeiträge epistemisch oder semantisch auf die eigenen bezogen sind. Anaphorische Beziehungen sind also prim a facie höchstens notwe ndige Bedingungen für die erfolgreiche und gehaltsteilende Kommunikation (indem durch sie zuvor nicht bestehende Rekurrenzstrukturen dynamisch im Austausch von Sprecher und Hörer hergestellt werden), aber keine $\mathrm{h}$ in re ichende $\mathrm{n}$ Bedingungen für die Sprecher und Hörer berechtigt verpflichtende gemeinsame korrekte Identifikation des semantischen Gehalts bestimmter anaphorischer Ketten.

Darum bedarf es für die Beurteilung der Frage, ob Brandoms semantische Theorie referenzfreie hinreichende Bedingungen für die Kommunikation mittels bestimmter Gehalte angibt und so ihr Ziel erreicht, eines genaueren Blicks auf die s e ma n t i s c h e Frage der notwendigen Bedingungen für die Unterscheidung anaphorischer Ketten für die Herstellung eines c o m m o $n$ t o $\mathrm{p}$ i c . Stellt sich hier heraus, dass bestimmende Unterscheidungen für Sprecher nur dann verfügbar sind, wenn man ihnen die Kompetenz zur Identifikation n ichtan a p hori s cher semantischer Bewertungen zuschreibt, dann kommt die inferentialistische Lösung des Kommunikationsproblems nicht ohne stillschweigend in Anspruch genommene autonome referenzielle Elemente aus. Dann aber ist zumindest die folgende nur mittels $(I N)$ folgende Einschätzung der Erklärungsreichweite von Brandoms Theorie nicht mehr haltbar: "truth and reference are philosopher's fictions, generated by grammatical misunderstandings" (MIE: 324). ${ }^{38}$ Die These (IN) ist keineswegs $\mathrm{h}$ a $\mathrm{r} \mathrm{m}$ l o s oder s e $\mathrm{m}$ a $\mathrm{n}$ ti s ch in ert. Ich möchte nun Gründe für die Gegenthese am Beispiel kontextsensitiver Interpretation illustrieren.

Für die Entwicklung dieser Gründe empfiehlt es sich, Brandoms Erklärung des Zustandekommens bestimm ter Gehalte anaphorischer Ketten zu betrachten. Wie gesehen, hat kommunikativer Erfolg (wie in §3, 150 ausgewiesen als "navigating perspectives in order to extract information from our remarks"; Brandom 2010b: 171) laut Brandom eine notwendige Bedingung: 
"extracting information from the remarks of others requires [...] to be able to tell what their beliefs would be true of if they were true" (MIE: 517). Wegen der perspektivenrelativen Natur substitutionsinferentiellen Gehalts verlangt kommunikativer Erfolg - das heißt die Bestimmung dessen als common topic in Bezug worauf doxastische Sprecherfestlegungen als Behauptungen formuliert sind - daher auch bei geteilter Sprache semantische Interpretation:

"Anaphoric connections [...] provide a way of mapping [...] different repertoires of substitutional commitments onto one another [...] to keep track of how each set of concomitant commitments relates to the others. [...] such correlation of the substitution-inferential commitments (and hence doxastic commitments) [...] is [...] interpretation that is the uptake by a scorekeeping audience of some speaker's claim" (MIE: 474f.).

Erfolgreiche Interpretation durch (stipulative) anaphorische Ko r rela t i o $n$ besteht also in der adäquaten und von beiden Gesprächteilnehmern anerkennbaren Identifikation einer be stim m te n (im Gegensatz zu anderen grammatisch erlaubten) anaphorischen Kette. Wäre allerdings der „uptake“, von dem Brandom hier spricht, nur a us d r u cks ge bunden in der Fortsetzung einer Kette durch Abbildung eines fremden Ausdrucks auf einen eigenen (dessen Signifikanz man selbst kennt) zu sehen, dann bliebe rätselhaft, wie sich dies als Vererbung von Gehalt oder Interp retation verstehen lässt, die es dem Hörer erlaubt, Information zu extrahieren. Daher ist der se mantis che Wert einer in der Beschreibung des Sprecherausdrucks reproduzie rbare anaphorischen Aufnahme nicht lediglich im Ausdruck zu sehen:

"the indirect description [...] only anaphorically refers to the specified token and so, as a whole, refers not to that token but rather to whatever that token (its anaphoric antecedent) refers to. [...] the referent of the whole description is to be understood not to be the term token $[\ldots]$ but rather [...] the referent of that antecedent token" (MIE: 311f.).

Insofern als überhaupt irgendwelche Information zur Extraktion kodiert ist, ist es dasjenige, worauf der Antezedenzausdruck bezogen ist. Nun kann letzterer natürlich seinerseits auf ein weiteres token bezogen sein und dieser wiederum und so weiter. Ließe sich diese Regression auf immer weitere Tokens nicht beenden, wäre somit laut Brandoms eigener notwendiger Bedingung kommunikativen Erfolgs ein solcher $\mathrm{n}$ i c h t möglich. We n n kommunikativer Erfolg im Sinne der Informationsextraktion aus Äußerungen also möglich ist, dann muss, für jede semantisch bestimmbare anaphorische Kette, ein letztes (beziehungsweise den Gehalt aller Kettenglieder standardisierendes) Glied des Verweises identifizierbar sein. Dieses Glied nennt Brandom „Initiator“ (MIE: 458). Anaphorische Ketten sind Rekurrenzstrukturen und 
"since recurrence and inheritance of substitutional commitments is transitive, so is anaphoric dependence. [...] anaphoric chains [...] are [...] anchored or initiated by tokenings that are not themselves anaphorically dependent on other tokenings" (MIE: 458).

Ohne diese Verankerung sind anaphorische Ketten nicht semantisch als Mengen individuierbar, die aus "members of one single token-repeatability structure" (MIE: 566) bestehen, das heißt nicht gehaltsbestimmt.

Anaphorische Abhängigkeit ist so als gehalts abhängige, das heißt se mantische Relation zu verstehen. Welche Bedeutung eine einzelne anaphorische Kette transportiert hängt von deren I nitiat or ab. Kommunikativer Erfolg wiederum hängt von der Fähigkeit von Sprechern zur B e s t i m mung des Gehalts der Gesamtkette und diese von der Fähigkeit der Unterscheidung des In itiators von allen anderen Gliedern der Kette ab. Somit besteht Brandoms eigenem Modell nach o h ne Initiatorbestimmung kein bestimmbarer Gehalt und somit kein kommunikativer Erfolg. Für die Frage, ob dieses Modell ohne referenzielle Annahmen auskommt, ist es wichtig, die Rolle dieser In itiatoren innerhalb der Semantik genauer zu fassen, um zu sehen, w e I c h e Normen für kommunikativen Erfolg verantwortlich zu machen sind.

Das erste Glied oder Einführungsereignis hat (wie auch in den nachfolgenden Zitaten ersichtlich) dreierlei Funktionen: (/1) Es nimmt Information auf, ist zugleich (I2) Te il der anaphorischen Kette, jedoch (I3) ein semantisch privilegiertes, da es den objektiven Standard für die Korrektheitsbedingungen aller weiteren Sätze bestimmt, die mithilfe der anaphorisch von ihm abhängigen tokens geformt werden.

Brandoms eigene Darstellung konzentriert sich in Kapitel 7 auf den Nachweis, dass die anaphorischen Rekurrenzstrukturen es erlauben, den Gehalt sogenannter „token-reflexives“ wie Demonstrativpronomen durch anaphorische Aufnahme weiteren Substitutionsschlüssen zugänglich zu machen (MIE: 458ff.). Damit sind sie für die Gehaltsbestimmung von Sätzen verarbeitbar, die unabhängig von der Äußerungssituation als Prämissen und Konklusionen dienen. Nur so kommen vom Bestehen einer Äußerungssituation abhängig bestimmte Gehalte unter die allgemeinen Normen semantischer Bewertung. Der entsprechende Nachweis steht unter dem Motto, dass Deixis Anapher präsupponiert. Für diesen gegen kausale Theorien der Gehaltsbestimmung gerichteten (und hier nicht bestrittenen) Nachweis ist einzig (I2) interessant. So heißt es "It is only as initiators of anaphoric chains [...] that [...] tokenings provide ways of talking or thinking about objects [...] These chains provide the point of using demonstratives [...] and they articulate the significance of doing so" (MIE: 573). Das bedeutet jedoch lediglich metasemantisch, dass das Bestehen irgendeiner Kette notwendige Bedingung der Gehalthaftigkeit von Demonstrativa und Indexikalien ist, und nicht semantisch ein Argument für die (seiner eigenen Darstellung nach widersinnige) Annahme, dass die Verfügung über die Kette hinreichend für die Bestimmung des Gehalts des Initiators sein könnte. 
Allerdings scheint Brandoms im engeren Sinne semantische These, dass die Hinzufügung des anaphorischen Apparats den Rahmen der rein inferentiellen Semantik nicht verlässt, davon abzuhängen, dass die durch (I3) in Bezug auf einen seinem eigenen Modell nach "not themselves anaphorically dependent on other tokenings" (MIE: 458) bestimmten Initiatoren a u ch - laut (I2) - Teil der Kette sind. Letztere wird dann gleitend aber ohne separate Rechtfertigung als in ihrer Gesamtheit (das heißt inklusive des Initiators) hinreichend (im Sinne eines deren Bezug bestimmenden Fregeschen Sinns) für die Bestimmung des Gehalts jedes einzelnen Kettenglieds (auch des Initiators) dargestellt. So heißt es (die gerade genannten (I1) und (I2) benennend):

"anaphorically structured constellations of tokenings [...] are like Fregean senses in that they determine the referents of the [...] tokenings whose significance they govern. [...] provide cognitive access to the particular objects [...] via intersubstitutability equivalence classes [...] which include demonstrative and indexical tokenings. Such chains anchor our thought and talk in particular talk it is about [...]. They determine [...] that we are talking and thinking about particular objects [and] what we are thereby talking and thinking about" (MIE: 592).

Hier klingt es - kontraintuitiv - so, als wenn aus dem Verweis auf die Gehaltsgleichheit alle r Kettenteile (als Äquivalenzklasse) folgen würde, dass sich der Gehalt auch des Initiators sozusagen aus der Kette retroaktiv ermitteln liesse (unabhängig vom Bestehen der Äußerungssituation und der Feststellung des in ihr vollzogenen referenziellen Erfolgs). Brandom vertauscht die (korrekte) Aussage, dass das Verfügen über die Kette (inklusive eines bereits in seinem semantischen Wert festgelegten Einführungsglieds ) zureichend für die Bestimmung des Gehalts jedes Kettenglieds ist mit der (seiner eigenen Darstellung der s e m a n t i s chen As y mmetrie anaphorischer Gehaltsbestimmung nach widersinnigen) Aussage, dass die Kette selbst es ist, was den Gehalt des Anfangsglieds bestimmt. ${ }^{39}$ Nur dann aber, wenn die z we ite Aussage zuträfe, wäre es gerechtfertigt, aus dem Bestehen anaphorischer Ketten auf deren in ferenti e II - s u b s t i t u tio nelle Natur zu schließen, denn dann wären ja tatsächlich sämtliche Normen zur Bestimmung von Gehalt der referenzanalogen anaphorischen Kettenglieder s e I b s t substitutioneller Natur, weil von der Verkettung her bis ins Initiativglied hinein eindeutig bestimmt. ${ }^{40}$

Doch genau dies wird an einer späteren Stelle als Irrtum erkennbar, an der Brandom in Übereinstimmung mit der semantischen Asymmetrie anaphorischer Gehaltsbestimmung ${ }^{41}$ erläutert, dass die objektive Normie rung der Kette (das heißt der für alle Sprecher verpflichtende Gehalt) wesentlich von der Identität des Initiators abhängt:

"To take one expression to be anaphorically dependent on another is to take it as inheriting its substitution-inferential role from the tokening that is its anaphoric antecedent. If you say 'That is a porcupine', and I pick up that premise and conclude '(so) it is a ver- 
tebrate', the truth of the conclusion I have drawn is to be settled (according to the interpreter) by what substitutions are appropriate (according to the interpreter) for the demonstrative tokening. [...] If (according to the interpreter) what the first speaker referred to by 'that' is the most cunning wooden replica of a porcupine [...], then, since this identity claim is to be understood as an intersubstitution license, [...] what I said is false" (MIE: 621; Hervorhebungen A.M.).

Entscheidend ist, dass der Gehalt der sprecherunabhängigen „intersubstitution-license“ anaphorischer Verkettung (und Äquivalenzklassenbildung) davon abhängt, worum es sich bei dem tatsächlich (sprecherunabhängig) gehandelt hat, worauf sich das Demonstrativum in der Einführungssituation bezogen hat. Denn was in der von Brandom beschriebenen Situation hö re r re l a t i v ist, ist ja lediglich die Identifikation dessen, worauf sich der Sprecher unter gegebenen Umständen bezogen hat. Die semantische Norm, dass dasjenige, was auch immer es gewesen ist, worauf sich der Sprecher mit dem Initiator letztlich bezogen hat, den Gehalt der restlichen Kettenglieder als den Gegenstand bestimmt, auf den sich das erste Glied bezogen hat ist demgegenüber $\mathrm{n}$ i c h t hörer- oder sprecherrelativ, da sie ja den Gehalt aller anaphorisch verknüpften tokens unabhängig davon asymmetrisch als gleich mit dem ersten Glied bestimmt, egal aus wessen Mund sie stammen. Es ist also nicht die Kette, sondern die Referenz des Initiators, die den sprecherneutral verpflichtenden und die Wahrheitsbedingungen der anaphorisch anknüpfenden Höreräußerung bestimmenden Gehalt der Äquivalenzklasse bestimmt. Das ist der g e h a I t s n o r m i e rende Sinn des Initiators nach (I3).

Zusammenfassend ergibt sich, dass Brandom zufolge die Fähigkeit von Sprechern zur Ausweisung von not anaphorically dependent aber gehaltsbestimmenden Initiativgliedern der Kette konstitutiv für die Bestimmbarkeit von extrahierbarer Information und damit kommunikativem Erfolg in anaphorisch aufeinander bezogenen Äußerungsfolgen ist. Schon allein aus dieser Bestimmung der semantischen Grundbegriffe folgt unmittelbar, dass es, da das Initiativglied gehalts bestimmend aber anapher unabhängig ist, Glieder der Kette gibt, die kommunikativen und (ko-) r e fe r e $n$ z individuierenden Gehalt besitzen, der nicht allein mittels der Verfügung über die Kette bestimmt ist. Brandoms Konstruktion der für die Gehaltsbestimmung anaphorisch generierter Äquivalenzklassen entscheidenden Rolle der Initiatoren zufolge wiederum wird an a pherunabhängig zugänglich gemachter (I2) Gehalt de facto als Beziehung von Gegenstand und Initiator in einer Verwendungssituation, (also Referenzherstellung) konzipiert, die die semantischen Normen für den korrekten uptake und Folgerung (also die Informationsextraktion) definiert. Sie ist explizit (durch die Asymmetrie der Substitutionsberechtigung) nicht inferentiell gerechtfertigt, sondern inferenzkorrektheitsnormierend. Aus dem Begriff des anaphorisch vererbten Gehalts ist das referenzfixierende Element als anaphernunabhängige semantische Eigenschaft der Kette (qua ihres Initiativglieds) n i cht e li m in i e rbar. Das impliziert, dass Brandoms 
These der rein inferentiellen Natur der Anapher $(I N)$ unzutreffend ist, da seiner eigenen Darstellung nach anaphernunabhängige aber gehaltsrelevante semantische Bestandteile anaphorischer Ketten konstitutiv für deren semantische Individuierung sind.

\section{Referenzabhängigkeit der Unterscheidung Initiator/Kettenglied am Beispiel anaphorischer Beziehungen auf kontextsensitive Äußerungen}

Es könnte nun so scheinen, als hinge das bislang entwickelte Argument gegen (IN) davon ab, dass es sich bei Brandoms Beispiel für die normierende Rolle von Initiatoren um ein Demonstrativum gehandelt hat und sich die augenscheinliche Irreduzibilität referenzieller Elemente bei der Gehaltsbestimmung anaphorischer Ketten durch eine infererenzialistische Auflösung demonstrativer Bezugnahme ${ }^{42}$ beseitigen und somit letztendlich doch als im Rahmen der inferentialistischen Erklärungsstrategie verständlich darstellen ließe. Dieser Anschein täuscht jedoch, denn dieselbe Unverzichtbarkeit der gerade geltend gemachten semantisch relevanten Fähigkeitsdualität, nämlich die anaphorisch-inferentiellen Kettenglieder und die referenziell-initiativen (privilegierten) Glieder voneinander zu unterscheiden, um den ve r e r b te $n$ Gehalt zu bestimmen, zeigt sich auch in Kontexten, in denen wir es mit Initiatoren zu tun haben, die nicht demonstrativ sind. Das ist zumindest der in der Pragmatik vorherrschenden linguistischen Standardtheorie der Anapher zufolge nicht überraschend, die für die nicht gebundenen Fälle anaphorischer Pronomen davon ausgeht, dass die semantischen Regeln es in einer großen Menge von Fällen offenlassen, ob diese Pronomen anaphorisch-abhängig oder in dexika I i s c h - referentiell zu interpretieren sind und somit Sprechern und Hörern die Fähigkeit $z u$ beiden Interpretationsarten zuschreiben sowie diejenige, um der Dialogkohärenz willen eine Unterscheidung beider treffen zu können (Neale 2006; Levinson 2000; Levinson 2006). ${ }^{43}$ Ein reprä-

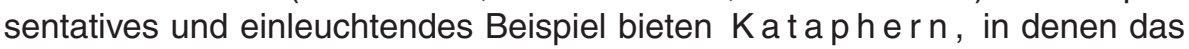
anaphorisch bindbare Pronomen vorangestellt ist, wie zum Beispiel in seine Frau liebt Herbert. Hier kann /seine/ als freies Demonstrativum dienen, um etwa anklagend auf den in der Sprechsituation anwesenden Kurt zu verweisen oder aber harmonisierend auf /Herbert/ vorzugreifen. In Brandoms Terminologie stehen hier also zwei anaphorische Ketten (mit durch subskribiertes $i$ indizierten Initiatoren und durch umklammertes $i$ indizierten gebundenen Vorkommnissen) offen, nämlich $\left\{/\right.$ seine $\left._{i}\right\}$ und $\left\{/\right.$ seine $_{(i)}, / /$ Herbert $\left._{i}\right\}$, deren erste das Gespräch auf den außerehelich geliebten Karl und deren zweite das Gespräch auf den ehelich geliebten Herbert lenkt. We I c he der beiden Ketten und somit w e Icher der beiden Gehalte als common to pic gelten muss, hängt davon ab, welche der beiden Interpretationen derselben tokens Sprecher und Hörer vornehmen. Um diese Unterscheidung zu treffen, müssen sie demnach zumindest die Möglichkeit haben, das referenzielle Vorkommnis von /seine/ als nicht anaphorisch gebunden (Initiator) zu identifizieren. Da in 
der Gesprächssituation keinerlei weitere Ketten verfügbar sind, aufgrund derer /seine/ mittels Koreferenz bestimmbar wäre, stellt diese Fähigkeit zur Gehaltsbestimmung die Befolgung einer nicht mit den Mitteln inferentiellsubstitutionell-anaphorischer Aspekte des Gehalts vorgenommene s e m a n tischen Norm dar.

Von Demonstrativa oder (laut Brandom ja inferentialistisch aufschlüsselbaren) deiktischen tokens unabhängige Beispiele für die zur Gehaltsbestimmung beziehungsweise Disambiguierung anaphorischer Ketten notwendigen Unterscheidung von Kettengliedern als (semantisch privilegierten) Initiatoren und abhängigen Gliedern ergeben sich aus Fällen von ko n t ex t s e n s it i ven oder syntaktisch inartikulierten Komponenten der semantischen Struktur geäußerter Behauptungssätze. Besonders der zweite Falltyp stellt eine Herausforderung für anapherzentrierte semantische Ansätze dar, da hier die anaphorisch abhängigen Kettenglieder auf $\mathrm{ke}$ in in der Performanz nachweisbares Token Bezug nehmen. Damit ist jedoch die Informationsextraktion nicht nur von einem "mapping of your tokens on mine" (s.o., §3; Brandom 2010b: 171), sondern auch von der gelegenheitsabhängigen Zuschreibung situativer Elemente zu Äußerungen abhängig, auf die dann anaphorisch nur deswegen Bezug genommen werden kann, weil die Ursprungsäußerung implizit referenziell determinierter ist, als es ihre Oberflächenform verrät.

Verdeutlicht werden kann dies an Fällen, in denen de r s e l b e A u s d ru ck gleich plausibel als Initiator als auch als anaphorisch abhängig aufgefasst werden kann.Um dies zu illustrieren, nehme ich folgenden Dialog zwischen Sigrid (später $S$ wie Sprecherin) und Herbert (oder $H$ wie Hörer) an, die sich über einen misslungenen Wanderurlaub ärgern.

Nehmen wir an, Sigrid sagt: „Es regnet schon wieder", und Herbert antwortet darauf mit: „Dieser Ort ist wirklich verflucht", und Sigrid bestätigt dies, indem sie sagt: „Ja, hier fahren wir nie wieder in den Urlaub hin. Jedes Mal, wenn wir in Bad Breckenreuth Urlaub machen, regnet es."Was Herbert mit einem unwirschen „Bad Idenhausen, nicht Breckenreuth“ kommentiert, worauf Sigrid mit „Ist doch egal, du weißt schon, was gemeint ist.", antwortet.

Wie gesehen, ergeben sich Unterschiede im Gehalt anaphorisch verketteter Ausdrucksereignisse dann, wenn zwei geäußerte tokens n i c h t k o r r e k t durcheinander ersetzbar sind. Die Inkorrektheit dieses Substitutionsschlußes ist dann gleichbedeutend damit, dass, gegeben zwei derartige tokens $/ A /$ und $/ B /$, token $/ B /$ nicht in die gleiche anaphorische Kette wie token / $A /$ eingegliedert werden darf. Worauf gründet nun die Korrektheit solcher Ersetzungsschlüsse? Der semantische Gehalt eines gegebenen tokens lässt sich durch seine Zugehörigkeit zu einer von Sprechern und Hörern unbestritten aufrechterhaltenen anaphorischen Kette charakterisieren. Diese sind wiederum raumzeitlich nicht unbestimmt, sondern den Sprechern praktisch verfügbare Realitäten, auf die sie mittels raumzeitlicher Verortung und bestimmter sprachlicher Mittel zugreifen können (wie anaphorisch indirekten bestimmten Beschreibungen (MIE: 305ff.) des Typs „was Sigrid (vorhin) als ,Bad Breckenreuth“ bezeichnet hat $\mathrm{s}_{1>}>$ was ich selbst (und alle anderen normalerweise) als ,Bad Brecken- 
reuth" bezeichne ${ }_{<t}>$ "). Der Nachweis der semantischen Unterschiedlichkeit zweier tokens hängt von der Nachweisbarkeit der Unterschiedlichkeit der sie enthaltenden Ketten ab, und diese wiederum von den E inführungsbedingungen der Kette und dem darin verwendeten ersten Glied oder Initiator. Können wir also Herbert und Sigrid $(H$ und $S$ ) als erfolgreich miteinander kommunizierend verstehen, so muss dies daran liegen, dass innen ausreichend semantische Normen für die Bestimmung dessen zur Verfügung stehen, was beiderseits als Gesprächsgegenstand erkennbar ist. Sehen wir von der Analyse des schon wieder ab, so lässt sich die im Bisherigen bereits vermutete Irreduzibilität referentieller Normen fü $r$ die Bestimmtheit der anaphorischen Ketten bestätigen.

Erstens bestätigt sich die Dualität referentieller und anaphorischer Rolle, denn H's / dieser Ort/ hätte grundsätzlich durch die Wahl des Demonstrativpronomens eine bevorzugte Interpretation als deiktische Bezugnahme auf den Sprecherort von $\mathrm{H}$. Es ist aber in diese m Kontext gerade nicht als deiktische, sondern präferent als anaphorische Bezugnahme auf den Sprecherort von S zu verstehen. Nur dann nimmt H's Äußerung auf irgendetwas Bezug, das $S$ gesagt hat und ist in diesem Sinne eine nicht-deviante Fortsetzung des Dialogs. Die Alternative ist jedoch nicht ausgeschlossen. Betrachtet $\mathrm{H}$ beispielsweise frustriert vom Regenwetter ein Foto von Venus auf dem Mobiltelefon und äußert /dieser Ort/ mit einer deiktischen Lesart, so handelt es sich um eine ebenfalls gelungene, aber ein neues The ma eröffnende Initiator-Äußerung. S muss also im Erfassen des von H Gesagten ein Vorkommnis des normalerweise deiktischen/dieserl als anaphorisch und nicht initiiere nd identifizieren können. ${ }^{44}$

Zweitens ist interessant, w i e der Ort von S in diesem Dialog erscheint, auf den sich H's Anknüpfung in der anaphorischen Lesart be zi e he n soll. Denn /dieser Ort/ nimmt ja n i c h t auf einen von S geäußerten A u s d r u ck Bezug. Wie ist dann aber ein ortserhaltender Rückbezug möglich? Die in der Literatur vorherrschende Erklärung (Stanley 2000; Stanley 2002; Stanley/Szabó 2000) postuliert versteckte (das heißt in der Äußerung nicht als Ausdrücke vorliegende) oder nichtartikulierte (Perry 2000: 181ff.; Perry/ Crimmins 2000: $221 \mathrm{ff}$. ${ }^{45}$ Parameter, die sich direkt als Verortungen des Sprechakts zum Beispiel auf die Interpretation indexikalischer oder kontextabhängig zu interpretierender Ausdrücke oder Ausdrucksweisen auswirken. Solche Parameter raumzeitliche Verortungen von tokens - sind sprachlicher Teil der Äußerung, aber nicht verbalisiert. Auch wenn umstritten ist, worum es sich bei solchen Parametern genau handelt (Teil der logischen Form? Pragmatische Komplemente?) und ob sie unvermeidlich oder optional sind ${ }^{46}$, ist ein guter Test für die semantische Realität solcher versteckter Parameter die Frage, ob sich sprachlich darüber quantifizieren lässt, das heißt ob ihre Werte als Werte von kontextuell verfügbaren aber nicht ausgedrückten Variablen darstellbar sind. Wenn Jedes Mal (...) in in S's Antwort von H als geglückt interpretiert wird und Bad Breckenreuth $\mathrm{n}$ i c h t erfolgreich als Ortsangabe ist, sondern nur als Platzhalter für eine solche, dann ist die Frage, welche Variable von Jedes Mal in (...) gebunden wird. Eine mögliche Antwort dar- 
auf ist die in Stanleys "binding argument" gegebene (Stanley 2000: 410ff.; Stanley/Szabo 2000: 243), dass Jedes Mal in einen Ortsparameter DA bindet, der in der Standardverwendung von Es regnet ausgedrückt, aber nicht verbalisiert ist. Er lässt sich allerdings durch Paraphrase leicht verbalisieren und die resultierenden Aussagen sich auf Synonymie mit der Quellaussage testen. Für das vorliegende Beispiel ergibt sich dann, dass Jedes Mal, wenn wir in Bad Breckenreuth im Urlaub sind, regnet es $d$ a komplett folgerungserhaltend gegenüber der unverbalisierten Form ist, wenn da als durch Jedes Mal gebunden aufgefasst wird. Für die Frage, ob referenzielle Normen bei der Bestimmung semantischen Gehalts eine Rolle spielen, ist die Beobachtung ausschlaggebend, dass H's /dieser Ort/ nicht a us drucks ab hängig und auch nicht übereine anaphorisch indirekte Beschreibung, sondern direkt auf einen Teil der Ausdrucksumstände von S als Sprecherort Bezug nimmt. Die raumzeitlichen Umstände der Äußerung von /es regnet/ werden dabei sozusagen $\mathrm{nachträglich} \mathrm{repräsentiert,} \mathrm{indem} \mathrm{ein} \mathrm{physischer,} \mathrm{beiden} \mathrm{verfüg-}$ barer Bezugspunkt als Initiator auszeichnet wird. Die entsprechende Kette ist dann als $\left\{/\right.$ Es regnet/ $\mathrm{DA}_{<S, x, y, z, t, t}$, /dieser Ort/ $\left.{ }_{(\mathrm{DA})}\right\}$ zu bestimmen, wobei dieser auf $D A<S, x, y, z, \downarrow$ direkt Bezug nimmt. Somit kann $\mathrm{H}$ nur dann an $\mathrm{S}$ anaphorisch anknüpfen, wenn $\mathrm{S}$ und $\mathrm{H}$ jeweils voraussetzen, dass/dieserOrt/ a Is e r folgreiche Bezugnahme auf den sprachlich nicht angesprochenen und damit lediglich existenziell verfügbaren Ort von $\mathrm{S}$ gelten kann. Anaphorische Verkettung ist in diesem Fall durch die Hinzunahme referenzieller Annahmen vermittelt, die eine Entscheidung zwischen den der stehenden Bedeutung von /dieser Ort/ gleichermaßen entsprechenden Bezugnahmen auf Sprecher- oder Hörerort herbeiführen.

Drittens hat auch S's Äußerung von hier mindestens zwei gleichermaßen berechtige Lesarten, eine anaphorische und eine demonstrativ-deiktische. ${ }^{47} \mathrm{~S}$ könnte sagen, dass sie nie wieder an den Ort fahren will, an dem sie gerade spricht oder, dass sie nie wieder an denselben Ort fahren will, der im Dialog verhandelt wird. Dass hier in einem entsprechenden Kontext wie dem obigen eine anaphorische Lesart hat, ist jedoch im Sinne der Unterscheidungen von Recanati (2004) o p ti o n a l. ${ }^{48} \mathrm{H}$ muss in seinem u p take also entscheiden, ob sich S's / hierl auf einen bestimmten Ort bezogen hat, der mit ihrem eigenen im kontextuell lizensierten Ausmaß so übereinstimmt, dass / hier/ im Sinne von am selben Ort, dem unsrigen (das heißt als / hier/ ${ }_{(\mathrm{DA})}$ ) interpretierbar ist oder aber eine potentiell neue Initialverortung vorschlagen will (das heißt /hier/, gemeint ist). Die Rationalisierung von S's Wahlverhalten lässt sich also nicht ohne ihr Wissen um den Unterschied zwischen anaphorischen und direkt-kontextinvolvierenden Bezugnahmen und somit we se nt lich se mantis che n Bezugnahmevoraussetzungen hinsichtlich der ihr verfügbaren Äußerungsausdrücke als korrekt beziehungsweise nach Regeln bestimmbar vorauszusetzen.

Viertens ist es semantisch problemlos anzunehmen, dass sich $\mathrm{S}$ und $\mathrm{H}$ auf ständig wechselnde beziehungsweise überhaupt keine Orte beziehen und die Menge $\left\{\left[\right.\right.$ Parameter DA $\left.A_{1}\right], \ldots$, /dieser Ort/.../hier/.../Bad Breckenreuth/ ... /Jedes $\mathrm{Mal}_{1}(x)\left[\right.$ Parameter $\left.D A_{x}\right] / \ldots / \neq$ Bad Breckenreuth/ .../[das,] was gemeint ist/\} 
keine anaphorische Kette darstellt. Alle Äußerungen lassen sich ja a u ch als Initiatoren auffassen. We $\mathrm{n}$ sie allerdings, was plausibel ist, irgendeine solche Kette darstellt, dann ist diese Kette erst dadurch individuiert als (zum Beispiel) ortsbezugserhaltend hinsichtlich Bad Idenhausen, dass sie durchsetzt ist mit Elementen, deren kontextabhängige Ausdruckssignifikanz für Substitutionsschlüsse sich den Sprechern und dem Semantiker n i c h t durch die anaphorische Kette allein erschließt, sondern nur durch die Verkettung der Ausdrucksäußerungen unter Voraussetzung darüber hinausgehender Mechanismen, die Bezugskontinuitätsvoraussetzungen auf Situationsaspekte fe s t legen.

Die Differenz zwischen Initiator und Glied der Kette geht somit für kein token aus der Kette selbst hervor, sondern nur aus einer Unterscheidung des Gebrauchssinnes der verkettbaren aber nicht notwendigerweise verketteten Äußerungsereignisse des spezifisch verwendeten Vokabulars. Wegen ihrer kontextabhängigen Unterdeterminierung müssen beispielsweise die hier-Äußerungen von Serst als Initiatoren festgelegt werden, selbst wenn sich $\mathrm{H}$ und $S$ beide in der für die de i kt i s c he Festlegung des Gehalts von hier inferentiell, epistemisch-normativ und kausal-raumzeitlich erforderlichen Lage befinden. Selbst wenn all diese Bedingungen positiv beschieden sind und Sigrid und Herbert beide wissen, dass dem so ist und einander alle erforderlichen epistemischen Berechtigungen erteilt haben (das heißt, das beiden und dem Theoretiker verfügbare inferentialistische Potential der Situation erschöpfend beschrieben ist), ist immer noch nicht klar, ob die von S vorgenommene Äußerung von / hier/ als auf H's /dieser Ort/(DA) und letztlich den Sprecherort von /es regnet $_{(\mathrm{DA})}$ bezogen und damit anaphorisch, oder aber als Neuinitiator deiktisch auf die eigenen Äußerungsumstände bezogen werden soll. Allein mit dem Repertoire von Anapher und Substitutionsfestlegungen lassen sich Herberts und Sigrids Unterscheidungen zwischen hier-Ereignissen als Initiatoren neuer Ketten und hier-Ereignissen als Gliedern einer bereits bestehenden Kette nicht erfassen. Da die Unterscheidung zwischen / hier/ INITIATOR $_{\text {und } / \text { hier/ }}$ (DA1) $\mathrm{im}$ Beispiel jedoch weder aus Unterschieden der Äußerung folgt (denn es ist ein und dieselbe Äußerung, die beide Lesarten zulässt) noch aus der Kette selbst hervorgeht (sondern nur aus H's Verständnis derselben als entweder neuinitiiert oder fortgesetzt), aber nach inferentialistischen Prinzipien selbst semantisch relavant ist (da Unterschiede in Initiatoren nach der anaphorisch-substitutionellen Semantik Unterschiede im Gehalt nach sich ziehen), gibt es für die inferentiell-substitutionell-anaphorische Semantik relevante Unterschiede, die Sprecher (und Theoretiker) nur dann machen können, wenn sie Inbezugsetzungen zwischen Äußerungsereignissen und Umständen zu interpretativen Zwecken zu verwenden in der Lage sind. Da hier nicht a u t o mati s ch im Sinne eines Index eine solche Inbezugsetzung leistet, ist das Erfolgen der Zuordnung prag mati s ch-n or mativer Natur und daher Symptom einer semantischen Norm. Daraus folgt, dass die praktische Fähigkeit des an a phorischen Anschlusses von H's an S's Bezugsrahmen und Ort von der Fähigkeit zur Bestimmung der s e mantis chen Interp retation abhängt, für die sich $\mathrm{H}$ oder S entscheiden sollten, die ihrerseits von der Fähig- 
keit zur Unterscheidung und Identifikation von Initiatoren als privilegierten Kettengliedern abhängt, die sich ihrerseits nur unter Zuhilfenahme nicht-anaphorischer semantischer Normen als Koordinierung irreduzibel referenzieller und inferentieller Normen erfüllen lässt. Damit ist der Inferentialismus allein entgegen Brandoms Anspruch $\mathrm{n}$ i c h $\mathrm{t}$ in der Lage, zureichende und referenzunabhängig formulierbare Bedingungen für die gehaltsbestimmte und - erhaltende kommunikative Sprachverwendung unter allen Umständen zu liefern.

\section{Schluss: Vieles bleibt offen}

Brandom beansprucht mit seiner Erweiterung des inferentialistischen Arsenals um subtitutionell-anaphorische und damit auf subsententielle Ausdrücke bezogene Elemente ein Äquivalent zu den von ihm abgelehnten repräsentationalistischen referenziellen Semantiken zu schaffen, das stark genug ist, um das aus dem Inferentialismus in seiner w e it e n Fassung resultierende Perspektivitäts- und Kommunikationsproblem zu lösen. Meine Folgerung aus den im Vorherigen entwickelten Argumenten ist, dass die Lösung des Kommunikationsproblems Brandoms eigenen Prämissen nach nu r am Erfolg des anaphorisch-substitutionellen Teils der Semantik hängt, weil semantische Beiträge der We it g e fa s s th e it des Inferenzbegriffs sie voraussetzen. Da sich die semantische Bestimmtheit der von Sprechern und Hörern miteinander in anaphorische Verkettungen gebrachten Ausdrücke jedoch wie gesehen nur dann erklären lässt, wenn diesen die Unterscheidung und An we ndbarkeit von Initiatoren, sowie der Unterscheidung von Initiatoren und anderen Kettengliedern zur Verfügung steht, und weil diese Unterscheidungen de facto referenziellen Annahmen äquivalent sind, ist Brandoms anaphorisch-substitutioneller Apparat entweder zur Lösung des Kommunikationsproblems ausreichend, aber dann nicht nur referenz a n a log (aber referenzfrei), sondern r e fe re n z i m plizierend und somit unvereinbar mit Brandoms offiziellem Antirepräsentationalismus. Alternativ sind anaphorische Verknüpfungen unter Aufrechterhaltung des antirepräsentationalistischen Vermeidungsprinzips referenzieller Grundannahmen referenzfrei zu konstruieren, dann aber u n b e s t im m t und somit keine Lösung des Kommunikationsproblems. Man könnte auch eine p a r tikularistische Strategie einschlagen und, obwohl semantische Wirksamkeit der Weitgefas s the it allge me in referenzfestlegende Voraussetzungen macht, solche Bestimmungen fallweise in Form von einzelnen materialen Festlegungen aufzulösen versuchen. ${ }^{49}$ Das Kommunikationsproblem bliebe dann wiederum nach Brandoms eigenen Prämissen ungelöst. Erstens sind die materialen Festlegungen perspektivenab hä ng i g und bestimmen damit lediglich perspektivenabhängige Sig n i fikanzen aber nicht perspektivenneutrale verpflichtende semantische Gehalte. Selbst wenn man solche als irgendwie praktisch verfügb a $r$ unterstellt, ergibt sich, wie gesehen, immer noch das Problem, dass jede neu hinzukommende einfache materiale Substitutionsfestlegung immer e n t - 
we de $r$ als Grund zur Veränderung von Überzeugungen ode $r$ als Veränderung semantischer Normen verstanden werden kann. Da die Entscheidung für Gemeinsamkeiten vorausgesetzt ist, weil die Identifikation der ri c h t i g e $\mathrm{n}$ (das heißt beiderseits verpflichtenden) Folgerung von dieser abhängt, erreicht man so nur Korrelation perspektivenabhängiger Vorentscheidungen, nicht aber ge me in s a me Normen. Bei dieser Strategie kreiste also, um Brandoms Navigationsmetapher zu verwenden, das Boot immer im eigenen Hafen. Wo Brandoms eigene Position in diesem Spektrum steht, ist erstaunlich schwer auszumachen. Als wenig hilfreich erwies sich Brandoms Verweis auf die We ite des Inferenzbegriffs im Sinne praktischer Eingangsund Ausgangsregeln. Die von Brandom übernommene Sellarssche Theorie des informationstragenden Wahrnehmungsaustauschs mit der Umgebung in Form verlässlicher Reaktionen auf Stimuli kann bestenfalls als Genealogie semantischer Normen durchgehen, die jedoch - wie Brandom selbst in Absetzung von naturalistischen Kausalsemantiken betont - ohne Erklärung der spezifisch inferentiellen und normativen Natur des Übergangs auf eine einfache Redeklarierung von kausalen in semantische Strukturen hinausläuft. Der weite Inferenzbegriff beseitigt hierbei lediglich die Frage des Zugangs zu Bezugsobjekten auf praktische Weise und eröffnet Sprechern und Hörern somit eine Ressource für die Lösung des Kommunikationsproblems, aber keine Lösung.

Brandoms offizieller Antirepräsentationalismus scheint das für die Lösung des Kommunikationsproblems erforderliche Zugeständnis eigenständiger referenzieller Normen nicht zu erlauben. In einer einschlägigen Diskussion mit Michael Kremer (2010) (und vielen mit McDowell (siehe Brandom 1996, McDowell 1997; Brandom 1997; McDowell 2008; Brandom 2008b)), der der Herkunft von bestimmten Umweltbezügen in Brandoms Theorie nachspürt und weder in dessen Theorie demonstrativer Bezugnahme noch in der Theorie des Bezugs singulärer Terme fündig wird, stellt Brandom selbst klar, dass er die Nichtauffindung von semantische Bestimmtheit aufklärenden etwa demonstrativen oder singulären Bezugnahmen für eine Bestätigung der Kohärenz seiner Theorie hält, der es ja gerade um deren Vermeid ung (zugunsten verlässlicher Gegenstandsunterscheidungsfähigkeiten in Wahrnehmung und Handlungsvollzügen) geht. Wie wichtig dieses Vermeidungsdesiderat für die Integrität seines Programms ist, geht wiederum an Stellen hervor, an denen die in der Diskussion mit Kremer als willkommen empfangene Unfähigkeit zur Auffindung effektiver Bezugsfestlegungsprämissen als theoretische Einsicht ausdrücklich gemacht wird. Brandom sieht den Verdienst seiner Theorie im deflationistisch-eliminativen ${ }^{50}$ Nachweis dafür, dass der herkömmlichen semantischen Theorien zugrundeliegende Begriff fixierter Referenz auf gemeinsame sprachunabhängige Objekte auf nichts weiter als einer hypostasierenden Verwechslung von dialogisch-grammatischen mit außersprachlich-objektabhängigen semantischen Strukturen beruht: "truth and reference are philosopher's fictions, generated by grammatical misunderstandings" (MIE: 324).

An anderen Stellen deutet Brandom eine in Diskussionen mit McDowell entwickelte konziliatorische Selbstdarstellung an, in der er die Plausibilität eines 
Mittelwegs zugesteht, der inferentialistisch und referenzialistisch identifizierbare Normen als irreduzibel aufeinander zugrundelegt und aufeinander als gegenseitige Beschränkungen bezieht. Als Hilfestellung zur korrekten Einordnung seiner Erklärung des "commitment to invert the representationalist order of explanation" (MIE: 135) gesteht Brandom bereits in MIE in einer Fußnote zu, dass er zwar den Gegensatz repräsentationalistischer und inferentialistischer Herangehensweisen betone, aber

"other possibilities include treating neither representation nor inference as explanatorily prior to the other. One might then go on to explain both in terms of some third notion, which is treated as more fundamental. Or one might eschew reductive explanations in semantics entirely" (MIE: 669). ${ }^{51}$

Auch in Antwort auf McDowells prinzipielle Einwände gegen den Versuch, begrifflichen Gehalt in Absehung von der Repräsentationsfunktion begrifflicher Ausdrucksmittel aufklären zu wollen (den McDowell als reduktionistisch versteht), macht Brandom wiederholt deutlich, dass

"[i]t may be, after all, that neither can be understood apart from the other - that reference and inference come as an indissoluble conceptual package that cannot be analyzed reductively, but only relationally. I agree, of course. Looking for a way to get an independent theoretical grip on one range of concepts, and then explicating the other in terms of it is only one strategy for illuminating the relations between the representational and inferential perspectives on semantic content. [...] The aim of MIE is not to say that the inferentialist order of explanation is the only one that can provide semantic illumination. It is to explore what kind of illumination it can provide [...] inference and representation are co-ordinate concepts" (Brandom 2008a: 215f.; Hervorhebung A.M.). ${ }^{52}$

Dies steht allerdings zu der offiziellen Auffassung in erheblicher Spannung. Allerdings täuscht es diese Stellen so auszulegen, als ob Brandom die r e fe renzunabhäng i ge Durchführung inferentialistischer Semantik als optional betrachte und man sie daher ohne Preisgabe des Erklärungsanspruchs bei Bedarf durch Hinzunahme referenzieller Elemente bereichern könnte. Denn Brandom beeilt sich, diese Zugeständnisse nicht als Preisgabe der inferentialistischen Erklärungsbasis misszuverstehen:

"But the aim of Chapters 5-8 of MIE is to say (to make explicit) in inferential terms what that representational dimension is. (...) By explicating the use of the representational terminology by means of the inferential terminology, we gain a better understanding" (Brandom 2008a: 217).

Wie gerade gesehen, ist dies zumindest hinsichtlich des Kommunikationsproblems unzutreffend, da es sich im Rahmen der n i ch t k on zilia to ris c h e n offiziellen semantischen Theorie nicht befriedigend lösen lässt.

Meiner Ansicht nach zeigen die zuvor gegen die Tragfähigkeit des anaphorisch-substitutionellen Teils der inferentiellen Semantik entwickelten Bedenken 
zusammen mit der Tatsache, dass weder die We ite des Inferenzbegriffs noch andere Ressourcen in Brandoms Theorie hinreichen, den von ihm selbst als Desiderat für die Erläuterung der Möglichkeit des perspektiventranszendierenden Informationsaustauschs identifizierten referenzanalogen Mechanismus adäquat zu charakterisieren, dass eine Lösung des Kommunikationsproblems im offizielle n inferentialistischen Rahmen nicht ansteht. Für einen gebrauchstheoretischen Ansatz wäre diese Nichteinholung der eigenen Basis jedoch ein ernstes Grundlagenproblem. Die in offizielle Strategie scheint deshalb attraktiver. Dass Referenz und Wahrheit Erfindungen von Philosophen sind, kann man dann allerdings nicht mehr behaupten. Sie werden so als genau die irreduzibel in Praktiken beanspruchten Normen erkennbar, die sie zu sein scheinen.

\section{Anmerkungen}

1 Dies wird insbesondere in seinem Austausch mit Fodor/Lepore $(2001 ; 2010)$ ersichtlich, in dem es Brandom gelingt, immer wieder aus atomistischem Standpunkt vorgetragene Einwände als bereits in MIE oder AR behandelte und in ihrer Lösung explizierte Problemstellungen zu identifizieren sowie ihre Lösung im Rahmen der inferentiell-substitutionell-anaphorischen Semantik zu benennen (siehe auch Brandom 2010a).

2 In meiner bewusst unscharfen Verwendung des Begriffs Gehalt schließe ich an Scharp (2012) an, demzufolge dasjenige, was die Aussage, der Satz, die Überzeugung und der Gedanke Johns gemeinsam haben, dass Gras grün ist, der(selbe) Gehalt ist. Diese Terminologie ist inzwischen in der Literatur zu propositionalen Einstellungen eingebürgert.

3 Siehe dazu auch die Beiträge von Prien und Harendarski in diesem Heft.

4 Dieser Gegensatz zum klassischen Pragmatismus spielt eine tragende Rolle in der Kritik an Brandom in Habermas (1999). Die entsprechenden Unterschiede zwischen Habermas und Brandoms Anschlüssen an den Pragmatismus sind gut zusammengefasst in Giovagnoli (2001).

5 Die hinter dieser Zielsetzung stehende Grundreaktion ist nicht neu. Brandoms Vermeidungsthese hat vielfältige auf referenz- oder wahrheitssemantischer Basis verfahrende externe Einwände gegen die Plausibilität der Theoriestrategie erzeugt (Taylor 2012). Eine weitere Richtung der Kritik folgt weitgehend den Einwänden (wie etwa bei Lycan (1986) oder Putnam (1988)) gegen die klassischen Formen der Begriffsrollensemantik (siehe Harman (1982), Greenberg/Harman (2006)). Ebenfalls extern, aber auf anderer Grundlage hat besonders McDowell wiederholt (zuletzt 2008) eine Ineliminierbarkeitsthese in Bezug auf demonstrative Bezugnahme auf Einzelgegenstände als Zugang zur Welt geltend gemacht, durch die semantische Bewertung erst sensitiv gegenüber und die Korrektheit gehaltvollen Denkens abhängig von Gegenständen wird. Angesichts von Brandoms enorm aufwendiger Rekonstruktion genau dieser Bezugnahmen im inferentialistischen Paradigma ist inm allerdings Recht damit zu geben, dass McDowells Beschwerde, dass Brandom keine Theorie eines (demonstrativen) Bezugs vorgelegt habe 
(McDowell 2008: 124) entweder heißt, dass a priori keine inferentialistische Theorie McDowells Anforderungen genügen würde oder aber achtlos über die in MIE in Sellars-Nachfolge vorgelegte Theorie der komplexen inferentiellen Verzahnungen von Umweltbedingungen und Sprecherverhaltensregelung hinweggeht. Bezüglich letzterem hat Brandom (2008b) in seine theoretischen Schritte und Leistungen nochmals in nützlicher Listenform wiedergegeben.

6 Zu einer verwandten Strategie aus Kantischer Perspektive siehe Kremer (2010).

7 Die Identifikation der Anapher als dem kommunikationstheoretischen Hauptstück in Brandoms Inferentialismus teilt mein Argument mit internen Kritikern. Diese weisen Brandoms eigene damit verbundene Begründungsansprüche für Objektivität als durch die lediglich interpersonell formatierten Anaphermechanismen und die dadurch ermöglichten Perspektivenverschränkungen in der doppelten Kont oführung gegenseitiger Gehaltszuschreibungen als unerfüllbar nach, das heißt: als unzureichend für die Lösung des Objektivitätsproblems (hierzu auch Prien 2010; Habermas 1999). Im Gegensatz zu diesen Untersuchungen geht es mir jedoch um die Frage, ob Brandoms Ressourcen für die dem Objektivitätsproblem seinem eigenen Ansatz nach vorgeordnete Erklärung der Möglichkeit von Kommunikation hinreichen.

8 In diesem Sinne weist auch Dohrn (2009: 461) in seiner überzeugenden Kritik an Brandoms inferentialistischen Auffassung von Eigennamen auf die Vereinbarkeit eines „schwach infererenzialistischen“ Standpunkts mit referenziellen Elementen hin.

9 Dies wird besonders in der Diskussion zwischen Brandom und Habermas deutlich. Siehe dazu auch erhellend Wanderer (2008: 168ff.) sowie Scharp (2012).

10 Vergleiche MIE: 588: "Communication is still possible, but it essentially involves intralinguistic interpretation - the capacity to accommodate differences in discursive perspective, to navigate across them", sowie MIE: 475: "Interpretation [...] is necessary even in the case where all parties share a language".

11 Vergleiche MIE: 474f.: "For information to be communicated is for the claims undertaken by one interlocutor to become available to others (who attribute them) as premises for inferences. Communication is the social production and consumption of reasons. [...] Anaphora plays an important role in securing the possibility of communication across the doxastic gap created by the differing commitments of speaker and audience. The capacity [...] of the audience to pick up a speaker's tokening anaphorically, and so connect it to their own substitution-inferential commitments is part of what makes it possible [...] (to extract) information from it".

12 Zum Beispiel MIE: 332: "Discursive practices incorporate actual things. [...] The way the world is constrains proprieties of inferential, doxastic, and practical commitment in a straightforward way from within those practices".

13 Dafür entwickelt Brandom als zusätzliche inferentialistische Ressource die auf der typischen Abwesenheit inkompatibler Festlegungen mit „unmittelbar erworbenen Festlegungen“ (das heißt Wahrnehmungsurteilen) aufbauenden „Zuverlässigkeitsschlüsse“. Die Schwierigkeiten, die in solchen Schlüssen vorgenommene Festlegung des Zuschreibenden auf die inferentiell relevante und den deontischen Status des so Festgelegten als typischerweise berechtigt, wenn festgelegt einzuschätzen nicht im Sinne einer referenziell bedingten Zuordnung von Wahrneh- 
mungssituation und zugeschriebener und unternommener Festlegung zu deuten, werden hervorragend verhandelt in Wanderer (2008: 189ff.).

14 Esfeld (2010) interpretiert Brandom folgerichtig dahingehend, dass er das Potential der Naturalisierung nicht ernst genug genommen habe, um ein Trilemma zu vermeiden, das sich mit dem Schluss von kausale auf normative Einschränkungen von Verhaltensregularitäten vermeiden ließe. Damit identifiziert Esfeld meiner Ansicht nach den Gedanken korrekt, der Brandom an diesen Stellen umtreibt; im Gegensatz zu Esfeld bin ich weniger optimistisch über Aussichten der Erläuterungen des Normativen aus dem Kausalen.

15 Brandom (2010b: 170f.): "Inferentialism and some of its Challenges".

16 Das Beispiel betrachtet auch Habermas (1999) kritisch. Prien (2010) führt es hingegen als erfolgreiche inferentialistische Erklärung und somit zusätzlich zur Verfügung stehende Erklärungsressource in Brandoms Sinne ins Feld.

17 Aus Normen kognitiver sensorischer Interaktion mit der Umwelt hervorgehende (zum Beispiel intentionale oder psychologische) Festlegungen von B e z u g n a h me $n$ können in Brandoms Ansatz wegen des Vermeidungsprinzips nicht direkt für die Erklärung von Korrekturpotentialen herangezogen werden, das heißt für die Erklärung der (zum Beispiel in an Putnam und Kripke anschließenden, aber auch in dynamischen Semantiken verhandelten) Möglichkeit, dass es zu die von Sprechern identifizierte (inferentielle) Semantik verändernden, aber deren semantischen Wert invariant lassenden Rückwirkungen der Ergebnisse der Anwendung von Allgemeinbegriffen auf Objekte durch die Objekte selbst kommen kann. Brandoms eigene Erklärung von Richtigstell ung en und der begrifflichen Objektivität ist entsprechend seiner inferentiellen Weichenstellungen erheblich komplizierter, scheinbar an schwerwiegende metaphysische Hintergrundannahmen gebunden (Habermas 1999) und auch in den Weiterentwicklungen in Brandom 2008a nicht befriedigend gelöst, wie Prien (2010), Gröbert (2009) und Whiting (2009) überzeugend nachweisen.

18 Russell bezeichnet solche Festlegungsakte kodifizierende Aussagen hilfreich als "truths in virtue of reference-determiner" (2008: 52ff.), um den Sonderstatus der entsprechenden doxastischen Festlegungen als zugleich semantische Verbindlichkeiten fixierend zu benennen.

19 Barth (2011) stellt diese Zusammenhänge in aller wünschenswerten Klarheit dar, folgt Brandom allerdings in der Auffassung, dass referenzielle Aspekte erst auf der rekonstruktiv-gewahrsammachenden Ebene erscheinen und für die Bestimmung der Richtigkeit einer Klassifizierungs r e a k t i o n keine Rolle spielen. Doch auch Barths Rekonstruktion gewinnt nur dadurch ihre Erklärungskraft, dass festliegt, was wodurch angereichert und nicht verändert wird und ist in diesem Sinne ebenfalls referenzinfiltriert.

20 Dem verwandte Probleme hervorragend herausarbeitenden Prien (2010: 454) unterläuft meiner Ansicht nach der Fehler, hier Brandoms Voraussetzung als Teil einer erfolgreichen inferentialistischen Erklärung (von Objektivität) heranzuziehen. Ich glaube mit Prien, dass die Interpretation und Rechtfertigung der Brandom-Sellarschen Formel, dass "the way the world is constrains proprieties [...] from within those practices" einer der entscheidenden Angelpunkte von Brandoms Programm darstellt (sowohl in Bezug auf das Objektivitätsproblem, das Prien ver- 
handelt, als auch in Bezug auf das Kommunikationsproblem, mit dem ich mich beschäftige), sehe aber anders als Prien hier eine Stelle, an der ohne nicht als inferentialistisch redeklarierbare, autonome referenzielle Grundannahmen der erwünschte epistemisch-praktische Erklärungserfolg nicht erzielbar ist.

21 Auf die Unterscheidung zwischen solchen re kognitive n Äusserung und wahrnehmungsreportierenden erstpersonalen Aussagen weisen Lance/Kukla (2010) hin: "In uttering it ['Lo, a rabbit'], I am making public various agent-neutral facts that can then be taken up as claims by others. [...] it is known - that there is a rabbit present. My agent-relative recognitive utterance passes on an agent-neutral entitlement." Von anderen übernimmt man dann nur den Bezug, nicht aber die Darstellungsbedingungen desselben.

22 Wie es in MIE: 208 heißt: "Appeal to regularity and irregularity can do normative explanatory work only if there is some way of privileging some regularities over others-some way, in other words, of saying what the correct regularity is".

23 Daher sehe ich solche Vorschläge wie den in Wolf (2006), eine referenzbasierte semantische Theorie mit Brandoms Inferentialismus zu vere in baren eher als Vorschläge an Brandom an, die Vermeidungsthese preiszugeben und zu einem schwachen Inferentialismus mit referenziellen Grundbegriffen überzugehen. Seine Beobachtungen identifizieren den inferentialistisch-anaphorischen Erklärungsgewinn in der Starrheit (die sich nach Brandoms überzeugender Darstellung in MIE: 468ff. besser als kommunikativ-anaphorisches als als historisch-kausales Verkettungsphänomen durch die Zeit verstehen lässt). Hier ist tatsächlich eine anaphorische Erklärung sehr fruchtbar, wie sich in alternativen Ansätzen der Erklärung von gegenüber variierenden doxastischen Hintergrundannahmen invarianter Referenz von durch Exemplifizierung eingeführten empirischen generellen Termini bei Berger (2002) und Ebbs (2007) erweist. Nichts davon stützt allerdings das Vermeidungsprinzip, da referenzielle Normierungen von Begriffen in diesen Ansätzen vorausgesetzt werden.

24 Wie verschiedene Kritiker Brandoms festgestellt haben, durchzieht das hier einschlägige Problem, wie sich aus zuverlässig erworbenen Unterscheidungsdispositionen eines gleichen Umwelteinflüssen ausgesetzten Kollektivs so etwas wie (Referenzersatz bietende) Normen der semantischen Korrektheit der Äußerungen jedes Mitglieds des Kollektivs ergeben können sollen, weite Teile von Brandoms spezifischer Form von Inferentialismus. Den wohl besten frühen Beitrag dazu hat Hattiangadi (2003) geleistet. Im Gegensatz dazu Loeffler (2005) und Esfeld (2011), die beide Brandom eine weitergehende Naturalisierung der Semantik empfehlen. Loeffler $(2008 ; 2014)$ nimmt hiervon allerdings zugunsten einer Kritik des Vermeidungsprinzips Abstand.

25 In einer Kritik der Begriffsrollensemantik Harmans im Anschluss an ähnliche Bedenken merkt Lycan (1986: 258) an, dass "I suspect that some people find CRS [(conceptual role semantics)] attractive only because they are tacitly infusing reference of some sort".

26 Es soll nicht der Eindruck entstehen, Brandom selbst irre sich über den Status der Substitutionsschlüsse. Sie dienen seiner eigenen Einschätzung nach weniger kommunikations- als kompetenzerklärenden Zielen, da sich so Kompositionalitätseffekte durch die Verfügbarkeit von Dekompositions- und Rekompositions- 
mechanismen erklären, während sich die Gesamtbedeutung nach wie vor inferentialistisch verstehen lässt. Vergleiche Brandom (2010, 174ff.).

27 MIE: 467: "Anaphora [...] is presupposed by [...] substitutional commitments".

28 Zur Typologie der Anaphern aus pragmatischer Sicht, in der dieser Art anaphorischen Rückbezugs eine wohldefinierte semantische Funktion zukommt. Siehe hierzu Huang 2004.

29 Es ist wichtig für Brandoms Ansatz, sein von vornherein pragmatisches Paradigma anaphorischer Abhängigkeit nicht mit dem in der Linguistik grundlegenden semantischen Modell der anaphorischen Bindungstheorie zu verwechseln. Zu diesen Unterscheidungen vergleiche Neale (2006); Levinson (2000, Kap. 4.3.2.5.).

30 MIE: 459: "It is crucial to the communicative function of anaphora that any term tokening [...] whatsoever can function as an anaphoric antecedent".

31 Dass es sich um allen Sprechern gleich verfügbare Basisfakten der Bedeutungsbeziehung handelt, sieht man zum Beispiel in MIE: 467: "Anaphora [...] is presupposed by [...] substitutional commitments. Taking one individual's tokening to be anaphorically dependent on another [...] is attributing a more primitive sort of commitment $[. .$.$] that determines which substitutional commitments [...] are relevant$ in assessing the substitutional significance. [...] Anaphorically related tokenings are coreferential." Hierzu auch MIE: 484ff.; 305ff.; beziehungsweise die Effekte einer öffentlichen Stipulation bei MIE:545. Besonders ausdrücklich in MIE: 460: "Anaphoric chains [...] are normative features [...] of the obligation that the significance assigned to [...] one part of the discourse answer in systematic ways to the significance assigned to [...] another." Hier ist die „obligation“ nicht abhängig von Perspektiven eingeführt.

32 MIE: 310: "The explicitly representational locutions by means of which we grasp and express the distinction between what our thought and talk is about and what we think and talk about it all depend on anaphoric mechanisms".

33 MIE: 467: "Taking one individual's token to be anaphorically dependent on another $[\ldots]$ is attributing a more primitive commitment, one that determines which substitutional commitments $[. .$.$] are relevant in assessing [...] significance".$

34 Auf diese wesentliche normative Zusatzbedingung (durch die eine perspektivenund einstellungstranszendente Normierung in Brandoms Theorie erst unabhängig eingeführt und als in der interpersonalen Grammatik korrekten anaphorischen Anschlusses an den Vorredner (das heißt dem I o g i s c h e $\mathrm{n}$, nicht dem s e m a nt i s ch e $n$ Teil des Phänomens) verortet a priori postuliert wird) und ihre fundamentale Rolle für den Erfolg von Brandoms Modell hat (allerdings ohne die hier vorgenommene Elaboration) ausdrücklich Loeffler (2005) hingewiesen.

35 Die Wichtigkeit des Postulats einer stipulativ eingeführten aber zugleich universal verpflichtenden semantischen Struktur für Brandoms Interpretation anaphorischer Phänomene hat Whiting (2008) identifiziert. Bave (2009: 60ff.) notiert die aus dem Postulatscharakter dieses Teils von Brandoms Modell resultierende Perplexität, weswegen Brandom dann nicht einfach explizit von Sprecher-Hörer-Einstellungen unabhängige In ter substitutivität von gewissen token-Ketten postuliert. Der Grund dafür ist natürlich, dass dies vielleicht de faciendo das ist, was an diesen Stellen in Brandoms Theoriebildung passiert, aber keinesfalls das 
ist, was passieren sollte, wenn keine extrainferentiellen semantischen Grundbegriffe erlaubt sind.

36 "The notion of anaphora that is appealed to [in the explanation] is [...] grammatically guaranteed coreference" (MIE: 285; Hervorhebung A.M.).

37 Daher ist es der Logik dieser Erklärung nach etwas irreführend, wenn Brandom in der Einleitung zu MIE über Kapitel 8 sagt: "that is where the representational dimension of discursive practice is explained in terms of the interaction of the social and the inferential articulation of the communication of reasons" (xxiii). Kapitel 8 fügt der bereits als erfolgt vorausgesetzten Erklärung der Möglichkeit der Kommunikation lediglich die Entfaltung des sozialen Charakters der Zuweisung tatsächlich (im Gegensatz zu perspektivenrelativ angenommenerweise) bindender deontischer Status hinzu, aber keine weitere Erklärung der "communication of reasons". Es sei denn, doch dies kann hier nicht weiterverfolgt werden, man ginge der Berechtigung zur Inanspruchnahme stipulierter anaphorischer Normen ihrerseits im Netzwerk der Status nach und vervollständigte damit eine dann allerdings undurchdringliche perspektivistische Z i r k u l a r i ä t .

38 Präsumptiv gemeint ist die Verwechslung der laut inferentiellen Natur (IN) des gram matischen Phänomens der Anapher mit von erfolgreich kommunizierenden Sprechern zusätzlich beanspruchbaren und für den Erfolg verantwortlichen, autonomen referenziellen semantischen Normen. Vergleiche die Brandomsche Erläuterung der explanatorischen Ordnung zwischen extraling u isti scher und intralinguistischer (anaphorischer) Referenz (MIE: 306f.).

39 Die entscheidende Äquivokation in Brandoms konstruktivem Nachweis des Zureichens anaphorischer Mechanismen für Referenzfunktionen hat Aikin (2010, Kap. 4.8) hervorragend mit Bezug auf Brandoms Analyse von Demonstrativa herausgearbeitet.

40 Diese Verwechslung resultiert aus der Betonung der anderweitig motivierten Fragestellung, nämlich wie gelegenheitsabhängige Äußerungsereignisse mit indexikalischen und demonstrativen Elementen überhaupt bedeutungsvoll werden können, deren Beantwortung (nur mittels anaphorisch generierter Rekurrenzstrukturen) dann zugleich als Beantwortung für die Frage genommen wird, wie (das heißt aufgrund welcher semantischer Normen) bestimmte anaphorische Ketten von anderen als semantisch gehaltsverschieden unterschieden werden können. Dass letztere Frage nicht bloß, wie Brandom hier und da wegwerfend bemerkt ("messy retail business" (MIE: 129f.); "with anaphora, it is helpful to put to one side the difficult psychological question of how scorekeepers in fact determine salience" (MIE: 461)), psychologisch-kognitiv, sondern konzeptionell entscheidend ist, ergibt sich aus der Rolle des Interpretationserfolgs in der referenzanalogen Praxis des diskursiven anaphorischen Rückbezugs innerhalb der Lösung des durch den Gehaltsperspektivismus entstandenen Kommunikationsproblems. Brandom kann also hier nicht entschuldigt werden oder auf eine Antwort verzichten.

41 Vergleiche die Definitionen bei MIE: 455.

42 Man könnte etwa Brandoms Erfolg der inferenzbasierten Darstellung RussellEvansscher e gozentrisch-indexikalis che r Bezugnahmen als "strong de re" (Kap. 7.IV. in Kombination mit Kap. 8.VI.) anführen.

43 Vergleiche auch die in Wolter (2009) diskutierten Fälle in den von Birner/Kaplan/ 
Ward (2007) diskutierten Spreizkonstruktionen und kopularen Demonstrativa wie in $A$ : Me? I never wallow. I suffer in silence. B: No, that's Christine gegenüber $A$ : Hey, that's your cousin who's sitting on the curb, isn't it? B: (In a coffee shop, to companion reading the newspaper) Hey, that's Christine!, in denen /that's Christine/ jeweils als entweder auf im gemeinsamen Kontextwissen bezugnehmende Anapher oder als initiierende Deixis aufgefasst werden kann (Wolter 2009: 462f.).

44 Wie kompliziert die Abhängigkeiten semantischen Gehalts von Äußerungsumständen tatsächlich sind, diskutiert Recanati (2007, Kap. 31ff.).

45 Dort heißt es dezidiert: "Unarticulated constituency and direct reference are of a single stripe".

46 Gegen Stanley's Auffassung, dass es sich um Mandate der logischen Form der Sätze handelt, argumentiert Recanati (2002; hierzu auch Recanati 2004, Kap. 7).

47 Die an Kaplans Unterscheidung zwischen reinen und demonstrativen Indexikalien anschliessende Diskussion der nicht-reinen aber dennoch indexikalischen Natur von hierfindet sich bei Predelli (1998a; 1998b), auf die Perry (2006) reagiert.

48 Recanati (2006) schliesst daraus sogar, dass anaphorische Pronomen grundsätzlich frei sind und Bind ungen besondere Festlegungen darstellen (das heißt nicht, wie bei Brandom, per se universell verbindlich sind).

49 Diese Strategie, schlägt Williams (2004) Brandom in Antwort auf Argumente vor, die kontextsensitive Elemente für irreduzibel referenzielle Normen heranführen.

50 Brandom in diese Richtung fertig zu denken, empfehlen zum Beispiel Loeffler (2005) sowie Price (2010).

51 Kremer (2010) misst dieser Fußnote große Bedeutung bei.

52 Replies in Stekeler-Weithofer (2008).

\section{Literatur}

Aikin, Scott (2010), Epistemology and the Regress Problem. New York: Routledge.

AR: Siehe Brandom 2000.

Barth, Christian und Holger Sturm (Hrsg.) (2011), Robert B. Brandoms Expressive Vernunft. Historische und systematische Untersuchungen. Paderborn: mentis.

Barth, Christian (2011), „Brandoms Expressivismus und die Konzeptualisierung impliziter Gehalte“. In: Christian Barth und Holger Sturm (Hrsg.), Robert B. Brandoms Expressive Vernunft. Historische und systematische Untersuchungen. Paderborn: mentis: $175-207$.

Båve, Arvid (2009), „A Deflationary Theory of Reference“. Synthese 169, 1: 51-73.

Berger, Alan (2002), Terms and Truth, Cambridge: MIT Press.

Birner, Betty J., Jeffrey P. Kaplan und Gregory Ward (2007), „Functional Compositiona-

lity and the Interaction of Discourse Constraints“. Language 83, 2: 317-43.

Brandom, Robert B. (1994), Making it explicit. Reasoning, Representing and Discursive Commitment. Cambridge, Mass.: Harvard University Press.

Brandom, Robert B. (1996), „Perception and Rational Constraint: McDowell's ,Mind and World“'. Philosophical Issues 7: 241-259.

Brandom, Robert B. (1997), „Replies“. Philosophy and Phenomenological Research 57, 1: 189-204. 
Brandom, Robert B. (2000), Articulating Reasons. An Introduction to Inferentialism. Cambridge, Mass.: Harvard University Press.

Brandom, Robert B. (2008a), Between Saying and Doing. Towards an Analytic Pragmatism. Oxford: Oxford University Press.

Brandom, Robert B. (2008b), „Responses“. In: Pirmin Stekeler-Weithofer (ed.), The Pragmatics of Making it Explicit. Amsterdam: John Benjamins Publishing: 209230.

Brandom, Robert B. (2010a), „Reply to Michael Kremer's ,Representation or Inference. Must We Choose? Should We?"“ In: Bernhard Weiss und Jeremy Wanderer (eds.), Reading Brandom: On Making it Explicit. Abingdon: Routledge: 347-352.

Brandom, Robert B. (2010b), „Inferentialism and Some of Its Challenges“. In: Bernhard Weiss und Jeremy Wanderer (eds.), Reading Brandom: On Making it Explicit. Abingdon: Routledge: 159-180.

BSD: Siehe Brandom 2008a.

Devitt, Michael und Richard Hanley (eds.) (2006), The Blackwell Guide to the Philosophy of Language. Oxford: Blackwell.

Dohrn, Daniel (2009), „Robert Brandom über singuläre Termini“. Zeitschrift für philosophische Forschung 63, 3: 453-465.

Dummett, Michael (1991), Frege and Other Philosophers. Oxford: Clarendon Press.

Ebbs, Gary (2009), Truth and Words. Oxford: OUP.

Esfeld, Michael und Christian Sachse (2010), Kausale Strukturen. Einheit und Vielfalt in der Natur und den Naturwissenschaften. Frankfurt am Main: Suhrkamp.

Esfeld, Michael (2011), „Brandoms Trilemma“. In: Christian Barth und Holger Sturm (Hrsg.), Robert Brandoms expressive Vernunft. Paderborn: mentis: 209-229.

Fodor, Jerry Alan und Ernie Lepore (2001), „Brandom's Burdens: A Review of Robert B. Brandom's Articulating Reasons“. Philosophy and Phenomenological Research 63: 465-82.

Fodor, Jerry Alan und Ernie Lepore (2010), „Brandom Beleaguered“. In: Bernhard Weiss und Jeremy Wanderer (eds.), Reading Brandom. On Making it Explicit. Abingdon: Routledge: 181-193.

Giovagnoli, Raffaela (2001), "On Normative Pragmatics: A Comparison Between Habermas and Brandom". Teorema 20, 3: 51-68.

Greenberg, Mark und Gilbert Harman (2006), „Conceptual Role Semantics“. In: Ernie Lepore und Barry Smith (eds.), The Oxford Handbook of Philosophy of Language. Oxford: OUP: 295-322.

Grönert, Peter (2008), „Brandom's solution to the objectivity problem“. In: Pirmin Stekeler-Weithofer (ed.), The Pragmatics of Making it Explicit. Amsterdam: John Benjamins Publishing: 147-162.

Habermas, Jürgen (1999), „Von Kant zu Hegel. Zu Robert Brandoms Sprachpragmatik“. In: Jürgen Habermas (Hrsg.), Wahrheit und Rechtfertigung. Philosophische Aufsätze. Frankfurt am Main: Suhrkamp: 138-184.

Harman, Gilbert (1982), „Conceptual Role Semantics“. Notre Dame Journal of Formal Logic 23, 2: 242-256.

Hattiangadi, Anandi (2003), „Making It Implicit: Brandom on Rule Following“. Philosophy and Phenomenological Research 66, 2: 419-431. 
Huang, Yan (2004), „Anaphora and the Pragmatics-Syntax Interface“. In: Laurence R. Horn und Gregory Ward (eds.), The Handbook of Pragmatics: 288-314.

Knell, Sebastian (2008), „A deflationist theory of intentionality?“ In: Pirmin StekelerWeithofer (ed.), The Pragmatics of Making it Explicit. Amsterdam: John Benjamins Publishing: 65-82.

Kremer, Michael (2010), „Representation or Inference: Must We Choose? Should We?“ In: Bernhard Weiss und Jeremy Wanderer (eds.), Reading Brandom. On Making it Explicit. Abingdon: Routledge: 227-246.

Lance, Mark und Rebecca Kukla (2010), „Perception, Language, and the First Person“. In: Bernhard Weiss und Jeremy Wanderer (eds.), Reading Brandom. On Making it Explicit. Abingdon: Routledge: 115-128.

Laurier, Daniel (2008), „Pragmatics, Pittsburgh style“. In: Pirmin Stekeler-Weithofer (ed.), The Pragmatics of Making it Explicit. Amsterdam: John Benjamins Publishing: 127-146.

Levinson, Stephen C. (2000), Presumptive Meanings. The Theory of Generalized Conversational Implicature. Cambridge Mass. und London: MIT Press.

Levinson, Stephen C. (2006), „Deixis“. In: Laurence L. Horn und Gregory Ward (eds.), The Handbook of Pragmatics. Oxford: Blackwell: 97-122.

Loeffler, Ronald (2005), „Normative Phenomenalism: On Robert Brandom's PracticeBased Explanation of Meaning“. European Journal of Philosophy 13, 1: 32-69.

Loeffler, Ronald (2009), „Neo-Pragmatist (Practice-Based) Theories of Meaning“. Philosophy Compass 4, 1: 197-218.

Loeffler, Ronald (2014), „Belief ascriptions and social externalism“. Philosophical Studies 168: 211-239.

Lycan, William G. (1986), „Semantics and Methodological Solipsism“. In: Ernie Lepore (ed.), Truth and Interpretation. Oxford: Blackwell: 245-261.

McDowell, John (1997), „Brandom on Representation and Inference“. Philosophy and Phenomenological Research 57, 1: 157-162.

McDowell, John (2008), „Motivating inferentialism: Comments on Making it Explicit (Ch. 2)“. In: Pirmin Stekeler-Weithofer (ed.), The Pragmatics of Making it Explicit. Amsterdam: John Benjamins Publishing: 109-126.

MIE: Siehe Brandom 1994.

Neale, Stephen (2006), „Pronouns and Anaphora“. In: Michael Devitt und Richard Hanley (eds.), The Blackwell Guide to the Philosophy of Language. Oxford: Blackwell: 335-373.

Perry, John (2000), „Thought without Representation“. In: John Perry (ed.), The Problem of the Essential Indexical and Other Essays. Stanford: CSLI Publications: 171188.

Perry, John (2006), „Using Indexicals“. In: Michael Devitt und Richard Hanley (eds.), The Blackwell Guide to the Philosophy of Language. Oxford: Blackwell: 314-334.

Perry, John und Mark Crimmins (2000), „The Prince and the Phone Booth: Reporting Puzzling Beliefs“. In: John Perry (ed.), The Problem of the Essential Indexical and Other Essays. Stanford: CSLI Publications: 207-232.

Predelli, Stefano (1998a), „Utterance, Interpretation and the Logic of Indexicals“. Mind and Language 13: 400-414. 
Predelli, Stefano (1998b), „I am not Here Now“. Analysis 58: 108-115.

Price, Huw (2010), „One Cheer for Representationalism?“ In: Randall E. Auxier und Lewis E. Hahn (eds.), The Philosophy of Richard Rorty. Chicago und La Salle: Open Court: 269-289.

Prien, Bernd (2010), „Robert Brandom on Communication, Reference and Objectivity“. International Journal of Philosophical Studies 18,3: 433-458.

Recanati, François (2002), „Unarticulated Constituents“. Linguistics and Philosophy 25, 3: 299-345.

Recanati, François (2004), Literal Meaning. Cambridge: Cambridge University Press.

Recanati, François (2006), „Deixis and Anaphora“. In: Zoltan G. Szabó (ed.), Semantics vs. Pragmatics. Oxford: Oxford University Press: 286-317.

Recanati, François (2007), Perspectival Thought. A Plea for (Moderate) Relativism. Oxford: Oxford University Press.

Russell, Gillian (2008), Truth in Virtue of Meaning, Oxford: Oxford University Press.

Scharp, Kevin (2012), „Brandom on Communication“. In: Jason Hannon und Robert Rutland (eds.), Philosophical Profiles in the Theory of Communication. Toronto: McGill-Queen's University Press: 99-126.

Stanley, Jason (2000), „Context and Logical Form“. Linguistics and Philosophy 23: 391434.

Stanley, Jason (2002), „Making it Articulated“. Mind and Language 17: 149-168.

Stanley, Jason und Zoltan G. Szabó (2000), „Quantifier Domain Restriction“. Mind and Language 15: 219-261.

Stekeler-Weithofer, Pirmin (ed.) (2008), The Pragmatics of Making it Explicit. Amsterdam: John Benjamins.

Taylor, Kenneth (2012), „Reference and Jazz Combo Theories of Meaning“. In: William P. Kabasenche, Michael O'Rourke und Matthew H. Slater (eds.), Reference and Referring. Cambridge: MIT Press: 271-304.

Wanderer, Jeremy (2008), Robert Brandom. Toronto: McGill-Queen's Press.

Weiss, Bernhard und Jeremy Wanderer (eds.) (2010), Reading Brandom. On Making it Explicit. Abingdon: Routledge.

Whiting, Daniel (2006), „Between Primitivism and Naturalism: Brandom's Theory of Meaning“. Acta Analytica 21, 3: 3-22.

Whiting, Daniel (2008), „Meaning Holism and De Re Ascription“. Canadian Journal of Philosophy 38: 575-599.

Williams, Michael (2004), „Context, Meaning, and Truth“. Philosophical Studies. An International Journal for Philosophy in the Analytic Tradition 117, 1-2: 107-129.

Wolf, Michael P. (2006), „Rigid Designation and Anaphoric Theories of Reference“. Philosophical Studies. An International Journal for Philosophy in the Analytic Tradition 130: 351-375.

Wolter, Linsey (2009), „Demonstratives in Philosophy and Linguistics“. Philosophy Compass 4, 3: 451-468. 
Prof. Dr. Axel Mueller

Department of Philosophy

Northwestern University

1860 Campus Drive, Crowe 1-141

Evanston IL 60208, USA

E-mail:muell@northwestern.edu 